\title{
Probabilistic approach to singular perturbations of viscosity solutions to nonlinear parabolic PDEs
}

\author{
Mingshang $\mathrm{Hu}^{*} \quad$ Falei Wang ${ }^{\dagger}$
}

\begin{abstract}
In this paper, we prove a convergence theorem for singular perturbations problems for a class of fully nonlinear parabolic partial differential equations (PDEs) with ergodic structures. The limit function is represented as the viscosity solution to a fully nonlinear degenerate PDEs. Our approach is mainly based on $G$-stochastic analysis argument. As a byproduct, we also establish the averaging principle for stochastic differential equations driven by $G$-Brownian motion $(G$ SDEs) with two time-scales. The results extend Khasminskii's averaging principle to nonlinear case.
\end{abstract}

Key words: singular perturbation, averaging principle, nonlinear PDE, $G$-Brownian motion MSC-classification: $60 \mathrm{H} 10,60 \mathrm{H} 30$

\section{Introduction}

The present paper is devoted to the research of singular perturbations for a class of fully nonlinear degenerate parabolic PDEs with ergodicity coefficients. Our main tool is the nonlinear stochastic analysis theory formulated by Peng [36]. Indeed, we shall investigate the singular perturbation problems through asymptotic analysis of SDEs with slow and fast time-scales in the $G$-expectation framework.

In this framework, Peng systemically established the nonlinear stochastic calculus theory, such as $G$-Brownian motion, $G$-stochastic integral and so on. Due to this nonlinear structure, the $G$ expectation theory provides a useful tool for the research of fully nonlinear PDEs and volatility ambiguity in finance. Indeed, Song [43] obtained gradient estimates for a class of fully nonlinear PDEs by coupling methods for $G$-diffusion processes, Biagini et al. [4] studied robust mean-variance hedging, and Fouque, Pun and Wong [16] considered the asset allocation problem among a risk-free asset and two risky assets with an ambiguous correlation between the two risky assets. A notion quite related to $G$-expectation is the second order BSDE (2BSDE) framework proposed by Soner, Touzi and Zhang [22]. Indeed, the setting of 2BSDE is more general than that of $G$-expectation, whereas $G$-expectation has more regularity, see [21, 31, 39, 41, 44] and the references therein for more research on this field.

\footnotetext{
*Zhongtai Securities Institute for Financial Studies, Shandong University. humingshang@sdu.edu.cn. Research supported by the National Natural Science Foundation of China (No. 11671231) and the Qilu Young Scholars Program of Shandong University.

${ }^{\dagger}$ Zhongtai Securities Institute for Financial Studies and School of Mathematics, Shandong University. flwang2011@gmail.com (Corresponding author). Research supported by the National Natural Science Foundation of China (No. 12031009, No. 11601282 and No. 11871310) and the Young Scholars Program of Shandong University. Hu and Wang's research was partially supported by the National Key R\&D Program of China (No. 2018YFA0703900).
} 
In the present article, we shall consider averaging principle for the following $G$-SDEs with rapidly varying coefficients: for each $x=(\tilde{x}, \bar{x}) \in \mathbb{R}^{n} \times \mathbb{R}^{n}$ and $0<\varepsilon<1$,

$$
\left\{\begin{aligned}
\widetilde{X}_{t}^{\varepsilon, x} & =\tilde{x}+\int_{0}^{t} \widetilde{b}\left(\widetilde{X}_{s}^{\varepsilon, x}, \bar{X}_{s}^{\varepsilon, x}\right) d s+\sum_{i, j=1}^{d} \int_{0}^{t} \widetilde{h}_{i j}\left(\widetilde{X}_{s}^{\varepsilon, x}, \bar{X}_{s}^{\varepsilon, x}\right) d\left\langle B^{i}, B^{j}\right\rangle_{s}+\int_{0}^{t} \widetilde{\sigma}\left(\widetilde{X}_{s}^{\varepsilon, x}, \bar{X}_{s}^{\varepsilon, x}\right) d B_{s} \\
\bar{X}_{t}^{\varepsilon, x} & =\bar{x}+\int_{0}^{t} \frac{\bar{b}\left(\widetilde{X}_{s}^{\varepsilon, x}, \bar{X}_{s}^{\varepsilon, x}\right)}{\varepsilon} d s+\sum_{i, j=1}^{d} \int_{0}^{t} \frac{\bar{h}_{i j}\left(\widetilde{X}_{s}^{\varepsilon, x}, \bar{X}_{s}^{\varepsilon, x}\right)}{\varepsilon} d\left\langle B^{i}, B^{j}\right\rangle_{s}+\int_{0}^{t} \frac{\bar{\sigma}\left(\widetilde{X}_{s}^{\varepsilon, x}, \bar{X}_{s}^{\varepsilon, x}\right)}{\sqrt{\varepsilon}} d B_{s}
\end{aligned}\right.
$$

where $\widetilde{b}, \bar{b}, \widetilde{h}_{i j}=\widetilde{h}_{j i}, \bar{h}_{i j}=\bar{h}_{j i}: \mathbb{R}^{2 n} \rightarrow \mathbb{R}^{n}, \widetilde{\sigma}, \bar{\sigma}: \mathbb{R}^{2 n} \rightarrow \mathbb{R}^{n \times d}$ are deterministic non-periodic functions. Here the parameter $\varepsilon$ is used to describe the ratio of time scale between the diffusion processes $\tilde{X}^{\varepsilon, x}$ and $\bar{X}^{\varepsilon, x}$. Then, with this time scale $\widetilde{X}^{\varepsilon, x}$ is referred as slow component and $\bar{X}^{\varepsilon, x}$ as the fast component, respectively. Note that the distribution of the slow component can be represented as the viscosity solution to the following fully nonlinear PDE (see section 2.2):

$$
\left\{\begin{array}{l}
\partial_{t} u^{\varepsilon}-G\left(\left(\sigma^{\varepsilon}\right)^{\top} D^{2} u^{\varepsilon} \sigma+2\left[\left\langle h_{i j}^{\varepsilon}, D u^{\varepsilon}\right\rangle\right]_{i, j=1}^{d}\right)-\left\langle b^{\varepsilon}, D u^{\varepsilon}\right\rangle=0, \quad(t, \tilde{x}, \bar{x}) \in(0, T) \times \mathbb{R}^{n} \times \mathbb{R}^{n} \\
u^{\varepsilon}(0, \tilde{x}, \bar{x})=\varphi(\tilde{x}),(\tilde{x}, \bar{x}) \in \mathbb{R}^{n} \times \mathbb{R}^{n} .
\end{array}\right.
$$

Then, our aim is to describe the limit behaviour of fully nonlinear PDE (2) as $\varepsilon \rightarrow 0$ through averaging of the $G$-SDEs (1) under some appropriate assumptions.

The averaging principle for SDEs was first studied by Khasminskii 29] in 1968. Under some ergodicity assumptions, Khasminskii proved that the slow diffusion process converges weakly to the so-called averaged SDE, whose coefficients are characterized by integrals with respect to the related invariant probability measure. Since then, the averaging principle for diffusion processes have been studied with great interest and moreover, it provides a powerful tool for the research of singular perturbation problems for linear parabolic PDEs. In particular, Khasminskii and Krylov [30] established the averaging principle for diffusion processes in non-ergodic case, and obtained the limit behavior of linear parabolic PDEs without ergodic coefficients. We refer the reader to [8, 17, 33, 35, 40] and the references therein for more research on this topic.

There is also a vast literature on the singular perturbations of nonlinear PDEs based on probabilistic argument. With the help of backward stochastic differential equations (BSDEs), Buckdahn and $\mathrm{Hu}$ [5] studied homogenization of viscosity solutions to semilinear parabolic PDEs with periodic structures, and Bahlali, Elouaflin and Pardoux [2, 3] extended the results of [30] to semilinear parabolic PDEs. In [7], Buckdahn and Ichihara considered homogenization of fully nonlinear parabolic PDEs in periodic case by stochastic control approach. For more research on this field, we refer the reader to $[6,12,20,34]$ and the references therein.

Motivated by the seminal work [29], we shall study the limit behaviour of the slow $G$-diffusion process in the ergodic case. However, due to the nonlinearity of $G$-expectation, the averaged $G$ SDE has more complicated structure, which cannot be described by averaging the coefficients of the slow component. In this case, the averaged coefficients will interact with each other, and cannot be identified separately (see Lemma 3.2). On the other hand, the invariant expectations may not coincide with the ergodic expectations in the $G$-expectation framework (cf. 23]). To overcome these difficulties, we shall combine nonlinear stochastic calculus and viscosity solution techniques to analyze the limit distribution of the slow component. In particular, we shall construct the averaged PDE through the ergodic BSDEs approach in the $G$-expectation framework. Indeed, the ergodic BSDEs introduced by Fuhrman, Hu and Tessitore [18] provides a useful method for the study of large time behaviour of parabolic PDEs (cf. [27, 28]).

For our purpose, we first give a priori estimate of the $G$-SDE (11) under the disspativity condition. This is crucial for the equicontinuity of the viscosity solutions to the PDEs (2) with fast varying 
coefficients. Then, according to the Arzelà-Ascoli theorem, we could build a convergent subsequence of the viscosity solutions. Finally, with the help of dynamic programming principle and Khasminskii's discretization approach, we show that the limit function is the unique viscosity solution to the averaged PDE, which is a fully nonlinear PDE independent of the argument $\bar{x}$. Moreover, in the spirit of the Markov property, we could obtain that the limit of finite dimensional distribution is also determined by the averaged PDE, which together with Kolmogorov's criterion for weak compactness implies Khasminskii's averaging principle. In addition, we also extend the $G$-SDEs (1) with two time-scales to a more general case.

In conclusion, we develop an alternative method for the research of averaging of SDEs and singular perturbations of fully nonlinear parabolic PDEs without periodic structures. In particular, we extend the one of 29] to a class of fully nonlinear PDEs through $G$-stochastic analysis methods. For a closest related research, we refer the reader to [1] and the references therein. In [1], Alvarez and Bardi used the so-called perturbed test function method to study more general fully nonlinear PDEs with periodic coefficients.

The paper is organized as follows. In section 2, we introduce the $G$-SDEs with slow and fast time-scales. Then, we state the main results in section 3. Section 4 is devoted to the proofs of two main theorems. In section 5 , we give an extended case.

\section{Formulation of the problem}

In this paper, for each Euclidian space, we denote by $\langle\cdot, \cdot\rangle$ and $|\cdot|$ its scalar product and the associated norm, respectively. For a given set of parameters $\alpha, C(\alpha)$ will denote a positive constant only depending on these parameters and may change from line to line.

\subsection{The Probabilistic Setup}

Let $\Omega=C_{0}^{d}\left(\mathbb{R}^{+}\right)$be the space of all $\mathbb{R}^{d}$-valued continuous paths $\left(\omega_{t}\right)_{t \geq 0}$ starting from origin, equipped with the locally uniform norm. For each $t \in[0, \infty)$, we define $\Omega_{t}:=\{\omega \cdot \wedge t: \omega \in \Omega\}$ and

$$
L_{i p}\left(\Omega_{t}\right):=\left\{\varphi\left(B_{t_{1}}, \ldots, B_{t_{k}}\right): k \in \mathbb{N}, t_{1}, \ldots, t_{k} \in[0, t], \varphi \in C_{b . l i p}\left(\mathbb{R}^{k \times d}\right)\right\}, \quad L_{i p}(\Omega):=\cup_{T \geq 0} L_{i p}\left(\Omega_{T}\right),
$$

where $C_{b . l i p}\left(\mathbb{R}^{k \times d}\right)$ denotes the space of all bounded and Lipschitz functions on $\mathbb{R}^{k \times d}$. Then, for each given monotonic and sublinear function $G: \mathbb{S}(d) \rightarrow \mathbb{R}$, Peng [36] constructed a sublinear expectation space $\left(\Omega, L_{i p}(\Omega), \hat{\mathbb{E}},\left(\hat{\mathbb{E}}_{t}\right)_{t \geq 0}\right)$, called $G$-expectation space, where $\mathbb{S}(d)$ denotes the space of all $d \times d$ symmetric matrices. The canonical process $B_{t}(\omega)=\omega_{t}$ is called $G$-Brownian motion.

Indeed, for each $\xi \in L_{i p}(\Omega)$ with the form of

$$
\xi=\varphi\left(B_{t_{1}}, B_{t_{2}}, \cdots, B_{t_{k}}\right), \quad 0=t_{0}<t_{1}<\cdots<t_{k} \leq T,
$$

and for each $t \in\left[t_{i-1}, t_{i}\right), i=1, \ldots, k$, we define the conditional $G$-expectation by

$$
\hat{\mathbb{E}}_{t}[\xi]:=u_{i}\left(t, B_{t} ; B_{t_{1}}, \cdots, B_{t_{i-1}}\right) .
$$

Here, the function $u_{i}\left(t, x ; x_{1}, \cdots, x_{i-1}\right)$ with parameters $\left(x_{1}, \cdots, x_{i-1}\right) \in \mathbb{R}^{(i-1) \times d}$ is the viscosity solution of the following $G$-heat equation:

$$
\partial_{t} u_{i}\left(t, x ; x_{1}, \cdots, x_{i-1}\right)+G\left(\partial_{x x}^{2} u_{i}\left(t, x ; x_{1}, \cdots, x_{i-1}\right)\right)=0, \quad(t, x) \in\left[t_{i-1}, t_{i}\right) \times \mathbb{R}^{d},
$$

with terminal conditions

$$
u_{i}\left(t_{i}, x ; x_{1}, \cdots, x_{i-1}\right)=u_{i+1}\left(t_{i}, x ; x_{1}, \cdots, x_{i-1}, x\right), \text { for } i<k,
$$


and $u_{k}\left(t_{k}, x ; x_{1}, \cdots, x_{k-1}\right)=\varphi\left(x_{1}, \cdots, x_{k-1}, x\right)$. The $G$-expectation of $\xi$ is defined by $\hat{\mathbb{E}}[\xi]=\hat{\mathbb{E}}_{0}[\xi]$.

For each $p \geq 1$, the completion of $L_{i p}(\Omega)$ under the norm $\|X\|_{L_{G}^{p}}:=\left(\hat{\mathbb{E}}\left[|X|^{p}\right]\right)^{1 / p}$ is denoted by $L_{G}^{p}(\Omega)$. Similarly, we can define $L_{G}^{p}\left(\Omega_{T}\right)$ for each fixed $T \geq 0$. In this paper, we always assume that $G$ is non-degenerate to ensure the well-posedness of $G$-BSDE (see appendix A), i.e., there exist two constants $0<\underline{\sigma}^{2} \leq \bar{\sigma}^{2}<\infty$ such that

$$
\frac{1}{2} \underline{\sigma}^{2} \operatorname{tr}[A-B] \leq G(A)-G(B) \leq \frac{1}{2} \bar{\sigma}^{2} \operatorname{tr}[A-B] \text { for } A \geq B .
$$

Theorem $2.1([\mathbf{1 3},[\mathbf{2 4}])$ There exists a weakly compact set $\mathcal{P}$ of probability measures on $(\Omega, \mathcal{B}(\Omega))$, such that

$$
\hat{\mathbb{E}}[\xi]=\sup _{P \in \mathcal{P}} E_{P}[\xi] \text { for all } \xi \in L_{G}^{1}(\Omega) .
$$

Now, we define capacity

$$
c(A):=\sup _{P \in \mathcal{P}} P(A), A \in \mathcal{B}(\Omega) .
$$

A set $A \in \mathcal{B}(\Omega)$ is polar if $c(A)=0$. A property holds quasi-surely (q.s.) if it holds outside a polar set. In what follows, we do not distinguish between two random variables $X$ and $Y$ if $X=Y$ q.s.

Definition 2.2 Let $M_{G}^{0}(0, T)$ be the collection of processes of the following form: for a given partition $\left\{t_{0}, \cdots, t_{N}\right\}$ of $[0, T]$

$$
\eta_{t}(\omega)=\sum_{i=0}^{N-1} \xi_{i}(\omega) I_{\left[t_{i}, t_{i+1}\right)}(t)
$$

where $\xi_{i} \in L_{i p}\left(\Omega_{t_{i}}\right), i=0,1,2, \cdots, N-1$. For each $p \geq 1$, denote by $M_{G}^{p}(0, T)$ the completion of $M_{G}^{0}(0, T)$ under the norm $\|\eta\|_{M_{G}^{p}}:=\left(\hat{\mathbb{E}}\left[\int_{0}^{T}\left|\eta_{t}\right|^{p} d t\right]\right)^{1 / p}$.

For simplicity, we denote by $\langle B\rangle:=\left(\left\langle B^{i}, B^{j}\right\rangle\right)_{i, j=1}^{d}$ the cross-variation process. Denote by $M_{G}^{p}\left(0, T ; \mathbb{R}^{d}\right)$ the set of $d$-dimensional stochastic process $\eta=\left(\eta^{1}, \cdots, \eta^{d}\right)$ such that $\eta^{i} \in M_{G}^{p}(0, T), i \leq d$. Similarly, we can define $L_{G}^{p}\left(\Omega ; \mathbb{R}^{d}\right)$. Then, for any $\eta \in M_{G}^{2}\left(0, T ; \mathbb{R}^{d}\right)$ and $\gamma \in M_{G}^{1}(0, T ; \mathbb{S}(d))$, the $G$-Itô integrals

$$
\int_{0}^{T} \eta_{s} d B_{s}:=\sum_{i=1}^{d} \int_{0}^{T} \eta^{i}(s) d B_{s}^{i} \text { and } \int_{0}^{T} \gamma_{s} d\langle B\rangle_{s}:=\sum_{i, j=1}^{d} \int_{0}^{T} \gamma_{i j}(s) d\left\langle B^{i}, B^{j}\right\rangle_{s}
$$

are well defined, see Peng [37, 38]. Moreover, we also have the corresponding $G$-Itô's calculus theory. The following inequalities will be used frequently in this paper.

Lemma $2.3\left([\mathbf{3 7},[38])\right.$ Assume that $\gamma \in M_{G}^{p}\left(0, T ; \mathbb{R}^{d}\right)$ and $\eta \in M_{G}^{p}(0, T ; \mathbb{S}(d))$. Then, for each $p \geq 2$, we have

$$
\begin{aligned}
& \hat{\mathbb{E}}\left[\sup _{t \in[0, T]}\left|\int_{0}^{t} \eta_{s} d B_{s}\right|^{p}\right] \leq C(p) \hat{\mathbb{E}}\left[\left(\int_{0}^{T}\left|\eta_{s}\right|^{2} d s\right)^{p / 2}\right], \\
& \hat{\mathbb{E}}\left[\left|\int_{0}^{T} \gamma_{s} d\langle B\rangle_{s}\right|^{p}\right] \leq C(p, T) \hat{\mathbb{E}}\left[\int_{0}^{T}\left|\gamma_{s}\right|^{p} d s\right] .
\end{aligned}
$$




\section{$2.2 \quad G$-SDE with two time-scales}

In this section, we shall state some basic results about $G$-SDE (1), which will be used in our subsequent discussions. Throughout this paper, each element $x \in \mathbb{R}^{2 n}$ is identified to $(\tilde{x}, \bar{x}) \in \mathbb{R}^{n} \times \mathbb{R}^{n}$, unless otherwise specified. We need the following assumption:

(H1) There exists a constant $L_{1}>0$ such that, for any $x, x^{\prime} \in \mathbb{R}^{2 n}$,

$$
\left|\ell(x)-\ell\left(x^{\prime}\right)\right| \leq L_{1}\left|x-x^{\prime}\right| \text { and }|\ell(0)| \leq L_{1} \text {, for } \ell=\widetilde{b}, \bar{b}, \widetilde{h}_{i j}, \bar{h}_{i j}, \widetilde{\sigma} \text { and } \bar{\sigma} .
$$

Under assumption (H1), the $G$-SDE (10) has a unique solution $\left(\widetilde{X}^{\varepsilon, x}, \bar{X}^{\varepsilon, x}\right) \in M_{G}^{2}\left(0, T ; \mathbb{R}^{2 n}\right)$ for each $T>0$ and we refer the reader to Chapter V in Peng [38] or Gao [19] for the proof. Then, for any $\varphi \in C\left(\mathbb{R}^{n}\right)$ of polynomial growth, we define the function

$$
u^{\varepsilon}(t, \tilde{x}, \bar{x}):=\hat{\mathbb{E}}\left[\varphi\left(\widetilde{X}_{t}^{\varepsilon, x}\right)\right], \forall x=(\tilde{x}, \bar{x}) \in \mathbb{R}^{n} \times \mathbb{R}^{n} .
$$

For convenience, set

$$
b^{\varepsilon}=\left[\begin{array}{c}
\widetilde{b} \\
\frac{\bar{b}}{\varepsilon}
\end{array}\right], h_{i j}^{\varepsilon}=\left[\begin{array}{c}
\widetilde{h}_{i j} \\
\frac{\bar{h}_{i j}}{\varepsilon}
\end{array}\right], \sigma^{\varepsilon}=\left[\begin{array}{c}
\widetilde{\sigma} \\
\frac{\sigma}{\sqrt{\varepsilon}}
\end{array}\right] .
$$

Then we have the following result.

Lemma 2.4 Suppose assumption (H1) holds. Then for each $T>0, u^{\varepsilon}$ is the unique viscosity solution of the following fully nonlinear PDEs:

$$
\left\{\begin{array}{l}
\partial_{t} u^{\varepsilon}-G\left(\left(\sigma^{\varepsilon}\right)^{\top} D^{2} u^{\varepsilon} \sigma+2\left[\left\langle h_{i j}^{\varepsilon}, D u^{\varepsilon}\right\rangle\right]_{i, j=1}^{d}\right)-\left\langle b^{\varepsilon}, D u^{\varepsilon}\right\rangle=0,(t, \tilde{x}, \bar{x}) \in(0, T) \times \mathbb{R}^{n} \times \mathbb{R}^{n}, \\
u^{\varepsilon}(0, \tilde{x}, \bar{x})=\varphi(\tilde{x}),(\tilde{x}, \bar{x}) \in \mathbb{R}^{n} \times \mathbb{R}^{n},
\end{array}\right.
$$

where $D u^{\varepsilon}=\left(\partial_{x_{i}} u^{\varepsilon}\right)_{i=1}^{2 n}$ and $D^{2} u^{\varepsilon}=\left[\partial_{x_{i} x_{j}}^{2} u^{\varepsilon}\right]_{i, j=1}^{2 n}$ for each $x=(\tilde{x}, \bar{x}) \in \mathbb{R}^{2 n}$. Moreover, it holds that

$$
u^{\varepsilon}(t, \tilde{x}, \bar{x})=\hat{\mathbb{E}}\left[u^{\varepsilon}\left(t-\delta, \widetilde{X}_{\delta}^{\varepsilon, x}, \bar{X}_{\delta}^{\varepsilon, x}\right)\right], \forall 0 \leq \delta \leq t \leq T .
$$

Proof. The proof follows from Theorem 5.3.7 of [38] or Theorem 4.5 in [22] and the fact that the $G$-SDE (11) is time-homogeneous.

From Lemma 2.4 we could study the asymptotic behavior of $u^{\varepsilon}$ as $\varepsilon \rightarrow 0$ through the slow component $\widetilde{X}_{t}^{\varepsilon, x}$. In the rest of the article, we are going to discuss the limit distribution of $\widetilde{X}^{\varepsilon, x}$ as $\varepsilon \rightarrow 0$.

Remark 2.5 The equation (3) is a fully nonlinear PDE without periodic structure, which is different from the existing research; see [1 3, 7, 29, 30, 33] and the references therein.

\section{The averaging principle}

This section is devoted to the research of limit behaviour of the slow $G$-diffusion process as $\varepsilon \rightarrow 0$. In order to describe the averaged PDE, we introduce the following auxiliary $G$-SDE: for any $x=(\tilde{x}, \bar{x}) \in$ $\mathbb{R}^{2 n}$

$$
\bar{X}_{t}^{x}=\bar{x}+\int_{0}^{t} \bar{b}\left(\tilde{x}, \bar{X}_{s}^{x}\right) d s+\sum_{i, j=1}^{d} \int_{0}^{t} \bar{h}_{i j}\left(\tilde{x}, \bar{X}_{s}^{x}\right) d\left\langle B^{i}, B^{j}\right\rangle_{s}+\int_{0}^{t} \bar{\sigma}\left(\tilde{x}, \bar{X}_{s}^{x}\right) d B_{s} .
$$

In what follows, we make use of the following assumptions. 
(H2) There exists a constant $\eta>0$ such that, for each $\tilde{x}, \bar{x}, \bar{x}^{\prime} \in \mathbb{R}^{n}$.

$$
\begin{aligned}
& G\left(\left(\bar{\sigma}(\tilde{x}, \bar{x})-\bar{\sigma}\left(\tilde{x}, \bar{x}^{\prime}\right)\right)^{\top}\left(\bar{\sigma}(\tilde{x}, \bar{x})-\bar{\sigma}\left(\tilde{x}, \bar{x}^{\prime}\right)\right)+2\left[\left\langle\bar{x}-\bar{x}^{\prime}, \bar{h}_{i j}(\tilde{x}, \bar{x})-\bar{h}_{i j}\left(\tilde{x}, \bar{x}^{\prime}\right)\right\rangle\right]_{i, j=1}^{d}\right) \\
& +\left\langle\bar{x}-\bar{x}^{\prime}, \bar{b}(\tilde{x}, \bar{x})-\bar{b}\left(\tilde{x}, \bar{x}^{\prime}\right)\right\rangle \leq-\eta\left|\bar{x}-\bar{x}^{\prime}\right|^{2}
\end{aligned}
$$

(H3) There exists a constant $L_{2}>0$ such that $|\ell(x)| \leq L_{2}(1+|\tilde{x}|)$ for $\ell=\widetilde{b}, \widetilde{h}_{i j}, \widetilde{\sigma}$ and $x=(\tilde{x}, \bar{x}) \in$ $\mathbb{R}^{2 n}$.

Remark 3.1 The assumption (H2) is called dissipativity condition, which ensures the ergodicity of the diffusion process $\bar{X}^{x}$ (cf. 10, 11, 18]). The assumption (H3) is equivalent to $|\ell(0, \bar{x})| \leq L_{2}$, which is used to establish a uniform moment estimate of order $p>2$ for the slow component $\widetilde{X}^{\varepsilon, x}$ (see Remark 4.6 in section 4).

Lemma 3.2 Suppose assumptions (H1)-(H3) are satisfied. Then, for each $(\tilde{x}, \bar{x}, p, A) \in \mathbb{R}^{2 n} \times \mathbb{R}^{n} \times$ $\mathbb{S}(n)$, the following limit

$$
\widetilde{G}(\tilde{x}, p, A):=\lim _{t \rightarrow \infty} \frac{1}{t} \hat{\mathbb{E}}\left[\int_{0}^{t}\left\langle p, \widetilde{b}\left(\tilde{x}, \bar{X}_{s}^{x}\right)\right\rangle d s+\sum_{i, j=1}^{d} \int_{0}^{t}\left(\left\langle p, \widetilde{h}_{i j}\left(\tilde{x}, \bar{X}_{s}^{x}\right)\right\rangle+\frac{1}{2} \widetilde{\sigma}_{i j}^{A}\left(\tilde{x}, \bar{X}_{s}^{x}\right)\right) d\left\langle B^{i}, B^{j}\right\rangle_{s}\right]
$$

exists and is independent of the argument $\bar{x}$, where the matrix $\widetilde{\sigma}^{A}=\left[\widetilde{\sigma}_{i j}^{A}\right]_{i, j}=\widetilde{\sigma}^{\top} A \widetilde{\sigma}$.

Proof. For each $\bar{x} \in \mathbb{R}^{n}$, consider the following ergodic $G$-BSDE: $\forall 0 \leq t \leq r<\infty$,

$$
\begin{aligned}
Y_{t}^{p, A, \bar{x}}= & Y_{r}^{p, A, \bar{x}}+\int_{t}^{r}\left(\left\langle p, \widetilde{b}\left(\tilde{x}, \bar{X}_{s}^{x}\right)\right\rangle-\widetilde{G}(\tilde{x}, p, A)\right) d s+\sum_{i, j=1}^{d} \int_{t}^{r}\left(\left\langle p, \widetilde{h}_{i j}\left(\tilde{x}, \bar{X}_{s}^{x}\right)\right\rangle+\frac{1}{2} \widetilde{\sigma}_{i j}^{A}\left(\tilde{x}, \bar{X}_{s}^{x}\right)\right) d\left\langle B^{i}, B^{j}\right\rangle_{s} \\
& -\int_{t}^{r} Z_{s}^{p, A, \bar{x}} d B_{s}-\left(K_{r}^{p, A, \bar{x}}-K_{t}^{p, A, \bar{x}}\right) .
\end{aligned}
$$

Under assumptions (H1) and (H3), it holds that $|\ell(\tilde{x}, 0)| \leq L_{1}(1+|\tilde{x}|)$ for $\ell=\bar{b}, \bar{h}_{i j}, \bar{\sigma}$, and

$$
\begin{aligned}
& \left|\langle p, \widetilde{b}(\tilde{x}, \bar{x})\rangle-\left\langle p, \widetilde{b}\left(\tilde{x}, \bar{x}^{\prime}\right)\right\rangle\right|+\sum_{i, j=1}^{d}\left|\left\langle p, \widetilde{h}_{i j}(\tilde{x}, \bar{x})\right\rangle-\left\langle p, \widetilde{h}_{i j}\left(\tilde{x}, \bar{x}^{\prime}\right)\right\rangle\right|+\frac{1}{2} \sum_{i, j=1}^{d}\left|\widetilde{\sigma}_{i j}^{A}(\tilde{x}, \bar{x})-\widetilde{\sigma}_{i j}^{A}\left(\tilde{x}, \bar{x}^{\prime}\right)\right| \\
& \leq C\left(L_{1}, L_{2}\right)(1+|\tilde{x}|)(|p|+|A|)\left|\bar{x}-\bar{x}^{\prime}\right| .
\end{aligned}
$$

Thus, by Lemma A.2 in appendix A, the above ergodic $G$-BSDE has a solution

$$
\left(Y^{p, A, \bar{x}}, Z^{p, A, \bar{x}}, K^{p, A, \bar{x}}, \widetilde{G}(\tilde{x}, p, A)\right) \in \mathfrak{S}_{G}^{2}(0, \infty) \times \mathbb{R} .
$$

Moreover, from Lemma A.3 in appendix A (taking $\kappa_{1}=L_{1}, \kappa_{2}=C\left(L_{1}, L_{2}\right)(1+|\tilde{x}|)(|p|+|A|)$ and $\left.\bar{\kappa}=L_{1}(1+|\tilde{x}|)\right)$, we have for each $t \in[0, \infty)$

$$
\begin{aligned}
& \left|\hat{\mathbb{E}}\left[\int_{0}^{t}\left\langle p, \widetilde{b}\left(\tilde{x}, \bar{X}_{s}^{x}\right)\right\rangle d s+\sum_{i, j=1}^{d} \int_{0}^{t}\left(\left\langle p, \widetilde{h}_{i j}\left(\tilde{x}, \bar{X}_{s}^{x}\right)\right\rangle+\frac{1}{2} \widetilde{\sigma}_{i j}^{A}\left(\tilde{x}, \bar{X}_{s}^{x}\right)\right) d\left\langle B^{i}, B^{j}\right\rangle_{s}\right]-\widetilde{G}(\tilde{x}, p, A) t\right| \\
& \leq C\left(L_{1}, \eta\right) C\left(L_{1}, L_{2}\right)(|p|+|A|)(1+|\tilde{x}|)\left(1+|\bar{x}|+L_{1}(1+|\tilde{x}|)\right) \\
& \leq C\left(L_{1}, L_{2}, \eta\right)(|p|+|A|)\left(1+|x|^{2}\right),
\end{aligned}
$$

which ends the proof.

Moreover, $\widetilde{G}(\tilde{x}, p, A)$ has the following properties. 
Lemma 3.3 Assume the conditions (H1)-(H3) hold. Then for each $\tilde{x}, \tilde{x}^{\prime}, p, p^{\prime} \in \mathbb{R}^{n}$ and $A, A^{\prime} \in \mathbb{S}(n)$,

(i) $\widetilde{G}\left(\tilde{x}, p+p^{\prime}, A+A^{\prime}\right) \leq \widetilde{G}(\tilde{x}, p, A)+\widetilde{G}\left(\tilde{x}, p^{\prime}, A^{\prime}\right)$,

(ii) $\widetilde{G}(\tilde{x}, \lambda p, \lambda A)=\lambda \widetilde{G}(\tilde{x}, p, A)$ for each $\lambda \geq 0$,

(iii) $\widetilde{G}(\tilde{x}, p, A) \geq \widetilde{G}\left(\tilde{x}, p, A^{\prime}\right)$, if $A \geq A^{\prime}$,

(iv) $\left|\widetilde{G}(\tilde{x}, p, A)-\widetilde{G}\left(\tilde{x}^{\prime}, p^{\prime}, A^{\prime}\right)\right| \leq C\left(L_{1}, L_{2}, \eta\right)\left(1+|\tilde{x}|^{2}+\left|\tilde{x}^{\prime}\right|^{2}\right)\left[(|p|+|A|)\left|\tilde{x}-\tilde{x}^{\prime}\right|+\left|p-p^{\prime}\right|+\left|A-A^{\prime}\right|\right]$. In particular, $\widetilde{G}$ is a continuous function.

Proof. We only prove Assertion (iv), since the others are obvious due to the sublinearity of $\hat{\mathbb{E}}$. Without loss of generality, assume that $\widetilde{h}_{i j}=0, i, j=1, \ldots, d$. Recalling Assertion (iii) of Lemma 4.3 in 25] (taking $\tilde{x}$ as the control argument), we obtain that

$$
\sup _{t \geq 0} \hat{\mathbb{E}}\left[\left|\bar{X}_{t}^{(\tilde{x}, \bar{x})}-\bar{X}_{t}^{\left(\tilde{x}^{\prime}, \bar{x}\right)}\right|^{2}\right] \leq C\left(L_{1}, \eta\right)\left|\tilde{x}-\tilde{x}^{\prime}\right|^{2}, \forall \bar{x} \in \mathbb{R}^{n} .
$$

It follows that

$$
\begin{aligned}
\hat{\mathbb{E}}\left[\left|\left\langle p, \widetilde{b}\left(\tilde{x}, \bar{X}_{s}^{(\tilde{x}, \bar{x})}\right)\right\rangle-\left\langle p^{\prime}, \widetilde{b}\left(\tilde{x}^{\prime}, \bar{X}_{s}^{\left(\tilde{x}^{\prime}, \bar{x}\right)}\right)\right\rangle\right|\right] & \leq|p| \hat{\mathbb{E}}\left[\left|\widetilde{b}\left(\tilde{x}, \bar{X}_{s}^{(\tilde{x}, \bar{x})}\right)-\widetilde{b}\left(\tilde{x}^{\prime}, \bar{X}_{s}^{\left(\tilde{x}^{\prime}, \bar{x}\right)}\right)\right|\right]+\left|p-p^{\prime}\right| \hat{\mathbb{E}}\left[\left|\widetilde{b}\left(\tilde{x}^{\prime}, \bar{X}_{s}^{\left(\tilde{x}^{\prime}, \bar{x}\right)}\right)\right|\right] \\
& \leq C\left(L_{1}, \eta\right)|p|\left|\tilde{x}-\tilde{x}^{\prime}\right|+L_{2}\left(1+\left|\tilde{x}^{\prime}\right|\right)\left|p-p^{\prime}\right|,
\end{aligned}
$$

where we have used the fact that $\left|\widetilde{b}\left(\tilde{x}^{\prime}, \bar{X}_{s}^{\left(\tilde{x}^{\prime}, \bar{x}\right)}\right)\right| \leq L_{2}\left(1+\left|\tilde{x}^{\prime}\right|\right.$ ) (see assumption (H3)) in the last inequality. By a similar analysis, we also deduce that

$$
\begin{aligned}
& \hat{\mathbb{E}}\left[\left|\tilde{\sigma}^{\top}\left(\tilde{x}, \bar{X}_{s}^{(\tilde{x}, \bar{x})}\right) A \widetilde{\sigma}\left(\tilde{x}, \bar{X}_{s}^{(\tilde{x}, \bar{x})}\right)-\tilde{\sigma}^{\top}\left(\tilde{x}^{\prime}, \bar{X}_{s}^{\left(\tilde{x}^{\prime}, \bar{x}\right)}\right) A^{\prime} \widetilde{\sigma}\left(\tilde{x}^{\prime}, \bar{X}_{s}^{\left(\tilde{x}^{\prime}, \bar{x}\right)}\right)\right|\right] \\
& \leq C\left(L_{1}, L_{2}, \eta\right)\left(1+|\tilde{x}|+\left|\tilde{x}^{\prime}\right|\right)|A|\left|\tilde{x}-\tilde{x}^{\prime}\right|+C\left(L_{2}\right)\left(1+\left|\tilde{x}^{\prime}\right|^{2}\right)\left|A-A^{\prime}\right| .
\end{aligned}
$$

Consequently, by the definition of $\widetilde{G}$ and Lemma 2.3 , we derive that

$$
\begin{aligned}
& \left|\widetilde{G}(\tilde{x}, p, A)-\widetilde{G}\left(\tilde{x}^{\prime}, p^{\prime}, A^{\prime}\right)\right| \leq \limsup _{T \rightarrow \infty} \frac{1}{T} \int_{0}^{T} \hat{\mathbb{E}}\left[\left|\left\langle p, \widetilde{b}\left(\tilde{x}, \bar{X}_{s}^{(\tilde{x}, \bar{x})}\right)\right\rangle-\left\langle p^{\prime}, \widetilde{b}\left(\tilde{x}^{\prime}, \bar{X}_{s}^{\left(\tilde{x}^{\prime}, \bar{x}\right)}\right)\right\rangle\right|\right] d s \\
& \quad+\limsup _{T \rightarrow \infty} \frac{1}{T} \hat{\mathbb{E}}\left[\left|\int_{0}^{T}\left(\tilde{\sigma}^{\top}\left(\tilde{x}, \bar{X}_{s}^{(\tilde{x}, \bar{x})}\right) A \widetilde{\sigma}\left(\tilde{x}, \bar{X}_{s}^{(\tilde{x}, \bar{x})}\right)-\tilde{\sigma}^{\top}\left(\tilde{x}^{\prime}, \bar{X}_{s}^{\left(\tilde{x}^{\prime}, \bar{x}\right)}\right) A^{\prime} \widetilde{\sigma}\left(\tilde{x}^{\prime}, \bar{X}_{s}^{\left(\tilde{x}^{\prime}, \bar{x}\right)}\right)\right) d\langle B\rangle_{s}\right|\right] \\
& \quad \leq C\left(L_{1}, L_{2}, \eta\right)\left(1+|\tilde{x}|^{2}+\left|\tilde{x}^{\prime}\right|^{2}\right)\left[(|p|+|A|)\left|\tilde{x}-\tilde{x}^{\prime}\right|+\left|p-p^{\prime}\right|+\left|A-A^{\prime}\right|\right],
\end{aligned}
$$

which is the desired result.

Next, we introduce the averaged PDE:

$$
\left\{\begin{array}{l}
\partial_{t} \widetilde{u}-\widetilde{G}\left(\tilde{x}, D \widetilde{u}, D^{2} \widetilde{u}\right)=0, \forall(t, \tilde{x}) \in(0, T) \times \mathbb{R}^{n}, \\
\widetilde{u}(0, \tilde{x})=\varphi(\tilde{x}), \forall \tilde{x} \in \mathbb{R}^{n},
\end{array}\right.
$$

where $\varphi \in C\left(\mathbb{R}^{n}\right)$ satisfies the polynomial growth condition. The above PDE has a unique viscosity solution $\widetilde{u}$ of polynomial growth (see Theorem 3.6). For the definition and basic properties of viscosity solution, we refer the reader to Crandall, Ishii and Lions [9]. 
Example 3.4 Assume that $G(A)=\frac{1}{2} \operatorname{tr}[A]$ and $\widetilde{h}_{i j}=\bar{h}_{i j}=0, i, j=1, \ldots, d$. Then, the $G$-Brownian motion reduces to a Brownian motion. Denote by

$$
\widetilde{b}(\tilde{x}):=\lim _{t \rightarrow \infty} \frac{1}{t} \hat{\mathbb{E}}\left[\int_{0}^{t} \widetilde{b}\left(\tilde{x}, \bar{X}_{s}^{x}\right) d s\right], \widetilde{a}(\tilde{x}):=\lim _{t \rightarrow \infty} \frac{1}{t} \hat{\mathbb{E}}\left[\int_{0}^{t} \widetilde{\sigma}\left(\tilde{x}, \bar{X}_{s}^{x}\right) \widetilde{\sigma}^{\top}\left(\tilde{x}, \bar{X}_{s}^{x}\right) d s\right], \forall \bar{x} \in \mathbb{R}^{n} .
$$

In this case, the corresponding generator function $\widetilde{G}$ is given by

$$
\widetilde{G}(\tilde{x}, p, A)=\langle p, \widetilde{b}(\tilde{x})\rangle+\frac{1}{2} \operatorname{tr}[\widetilde{a}(\tilde{x}) A], \forall(\tilde{x}, p, A) \in \mathbb{R}^{n} \times \mathbb{R}^{n} \times \mathbb{S}(n) .
$$

Under some appropriate conditions, Khasminskii [29] proved that $u^{\varepsilon}(t, \tilde{x}, \bar{x})$ converges to $\widetilde{u}(t, \tilde{x})$ through the martingale problem approach. Moreover, $\widetilde{X}_{t}^{\varepsilon, x}$ converges in law to $\widetilde{X}_{t}^{\tilde{x}}$, where

$$
\tilde{X}_{t}^{\tilde{x}}=\tilde{x}+\int_{0}^{t} \widetilde{b}\left(\tilde{X}_{s}^{\tilde{x}}\right) d s+\int_{0}^{t} \sqrt{\widetilde{a}}\left(\widetilde{X}_{s}^{\tilde{x}}\right) d W_{s}
$$

Here $\sqrt{\widetilde{a}}$ is a square root of the $n \times n$ matrix $\widetilde{a}$ and $W$ is a $n$-dimensional Brownian motion.

Example 3.5 Suppose that all the coefficients of $G$-SDE are independent of the slow component $\widetilde{X}^{\varepsilon, x}$, and $\widetilde{h}_{i j}=\bar{h}_{i j}=0, i, j=1, \ldots, d$. Then, the $G$-SDE (1) reduces to

$$
\widetilde{X}_{t}^{\varepsilon, x}=\tilde{x}+\int_{0}^{t} \widetilde{b}\left(\bar{X}_{s}^{\varepsilon, x}\right) d s+\int_{0}^{t} \widetilde{\sigma}\left(\bar{X}_{s}^{\varepsilon, x}\right) d B_{s}, \quad \bar{X}_{t}^{\varepsilon, x}=\bar{x}+\int_{0}^{t} \frac{\bar{b}\left(\bar{X}_{s}^{\varepsilon, x}\right)}{\varepsilon} d s+\int_{0}^{t} \frac{\bar{\sigma}\left(\bar{X}_{s}^{\varepsilon, x}\right)}{\sqrt{\varepsilon}} d B_{s} .
$$

Furthermore, assume that $\bar{b}(0)=0$ and $\bar{\sigma}(0)=0$. It is obvious that $\bar{X}_{t}^{\varepsilon, 0}=\bar{X}_{t}^{0}=0$ for each $\varepsilon \in(0,1)$. Thus, from Assertion (ii) of Lemma A.1 in appendix A, we obtain that

$$
\hat{\mathbb{E}}\left[\left|\bar{X}_{t}^{\varepsilon, x}\right|^{2}\right] \leq \exp \left(-\frac{2 \eta t}{\varepsilon}\right)|\bar{x}|^{2}, \forall t \geq 0 .
$$

It follows that

$$
\hat{\mathbb{E}}\left[\left|\widetilde{X}_{t}^{\varepsilon, x}-\tilde{x}-\widetilde{b}(0) t-\widetilde{\sigma}(0) B_{t}\right|\right] \leq C\left(L_{1}, t\right)\left(\hat{\mathbb{E}}\left[\int_{0}^{t}\left|\bar{X}_{s}^{\varepsilon, x}\right|^{2} d s\right]\right)^{\frac{1}{2}} \leq C\left(L_{1}, \eta, t\right)|\bar{x}| \sqrt{\varepsilon},
$$

which implies that $\widetilde{X}_{t}^{\varepsilon, x}$ converges to $\tilde{x}+\widetilde{b}(0) t+\widetilde{\sigma}(0) B_{t}$ in $L_{G}^{1}$-norm.

According to Lemma 2.4, we can derive that the function

$$
\widetilde{u}(t, \tilde{x})=\hat{\mathbb{E}}\left[\varphi\left(\tilde{x}+\widetilde{b}(0) t+\widetilde{\sigma}(0) B_{t}\right)\right]
$$

is the unique viscosity solution to the averaged PDE (9) with generator

$$
\widetilde{G}(p, A)=\lim _{t \rightarrow \infty} \frac{1}{t} \hat{\mathbb{E}}\left[\int_{0}^{t}\left\langle p, \widetilde{b}\left(\bar{X}_{s}^{0}\right)\right\rangle d s+\frac{1}{2} \int_{0}^{T} \widetilde{\sigma}^{A}\left(\bar{X}_{s}^{0}\right) d\langle B\rangle_{s}\right]=\langle p, \widetilde{b}(0)\rangle+G\left(\widetilde{\sigma}^{A}(0)\right) .
$$

Now, we are in a position to state the main results.

Theorem 3.6 Suppose assumptions (H1)-(H3) hold. Then, for each $\varphi \in C\left(\mathbb{R}^{n}\right)$ of polynomial growth, the averaged PDE (9) admits a unique viscosity solution $\widetilde{u}$ satisfying the polynomial growth condition, and

$$
\lim _{\varepsilon \rightarrow 0} u^{\varepsilon}(t, \tilde{x}, \bar{x})=\widetilde{u}(t, \tilde{x}), \forall(t, \tilde{x}, \bar{x}) \in[0, \infty) \times \mathbb{R}^{2 n} .
$$


The proof of Theorem 3.6 will be given in section 4. The following result is a direct consequence of Theorem 3.6

Corollary 3.7 Suppose all the assumptions of Theorem 3.6 hold. Then, for each $(t, x) \in[0, \infty) \times \mathbb{R}^{2 n}$ with $x=(\tilde{x}, \bar{x})$, the slow $G$-diffusion process $\widetilde{X}_{t}^{\varepsilon, x}$ converges in law as $\varepsilon \rightarrow 0$, i.e.,

$$
\lim _{\varepsilon \rightarrow 0} \hat{\mathbb{E}}\left[\varphi\left(\widetilde{X}_{t}^{\varepsilon, x}\right)\right]=\widetilde{u}(t, \tilde{x}) .
$$

The Corollary 3.7 indicates that the distribution of the slow component can be approximated by the solution to the averaged PDE (9), which is independent of the argument $\bar{x}$.

Example 3.8 Consider the $G$-SDE (10). Assume that $n=d=1$ and $\widetilde{\sigma} \equiv 0$. In this case, the generator $\widetilde{G}$ is given by

$$
\widetilde{G}(p)=p^{+} \lim _{t \rightarrow \infty} \frac{1}{t} \hat{\mathbb{E}}\left[\int_{0}^{t} \widetilde{b}\left(\bar{X}_{s}^{x}\right) d s\right]+p^{-} \lim _{t \rightarrow \infty} \frac{1}{t} \hat{\mathbb{E}}\left[-\int_{0}^{t} \widetilde{b}\left(\bar{X}_{s}^{x}\right) d s\right]=: \bar{\mu} p^{+}-\underline{\mu} p^{-} .
$$

Then, from Proposition 2.2.7 of [38], there exists a maximally distributed random variable $\widetilde{\zeta}$, such that the function

$$
\widetilde{u}(t, \tilde{x}):=\hat{\mathbb{E}}[\varphi(\tilde{x}+t \widetilde{\zeta})]=\max _{\underline{\mu} \leq r \leq \bar{u}} \varphi(\tilde{x}+r t)
$$

is the unique viscosity solution to the following PDE:

$$
\left\{\begin{array}{l}
\partial_{t} \widetilde{u}-\widetilde{G}(D \widetilde{u})=0, \forall(t, \tilde{x}) \in(0, T) \times \mathbb{R}^{n}, \\
\widetilde{u}(0, \tilde{x})=\varphi(\tilde{x}), \forall \tilde{x} \in \mathbb{R}^{n} .
\end{array}\right.
$$

By Theorem 3.6. we deduce that $\widetilde{X}_{t}^{\varepsilon, x}=\tilde{x}+\int_{0}^{t} \widetilde{b}\left(\bar{X}_{s}^{\varepsilon, x}\right) d s$ converges in law to the maximal distribution $\tilde{x}+t \widetilde{\zeta}$ as $\varepsilon \rightarrow 0$, which can be seen as the law of large number for $G$-diffusion process. Therefore, we usually cannot obtain the pointwise convergence of $\widetilde{X}_{t}^{\varepsilon, x}$ (cf. [15]), which is different from the linear case (cf. [17]).

Moreover, with the help of Markov property for $G$-SDEs, we can also deal with the finite dimensional distribution of the slow $G$-diffusion process.

Theorem 3.9 Assume that (H1)-(H3) hold. Then, for each $x \in \mathbb{R}^{2 n}$ and $\varphi \in C\left(\mathbb{R}^{k \times n}\right)$ of polynomial growth, we have, for any $0 \leq t_{1} \leq t_{2}<\cdots t_{k}<\infty$,

$$
\lim _{\varepsilon \rightarrow 0} \hat{\mathbb{E}}\left[\varphi\left(\widetilde{X}_{t_{1}}^{\varepsilon, x}, \widetilde{X}_{t_{2}}^{\varepsilon, x}, \cdots, \widetilde{X}_{t_{k}}^{\varepsilon, x}\right)\right]=\lim _{\varepsilon \rightarrow 0} \hat{\mathbb{E}}\left[\varphi^{k-1}\left(\widetilde{X}_{t_{1}}^{\varepsilon, x}\right)\right],
$$

where $\varphi^{k-1}$ is defined iteratively through

$$
\begin{aligned}
\varphi^{1}\left(\tilde{x}^{1}, \tilde{x}^{2}, \cdots \tilde{x}^{k-1}\right)= & \lim _{\varepsilon \rightarrow 0} \hat{\mathbb{E}}\left[\varphi\left(\tilde{x}^{1}, \tilde{x}^{2}, \cdots, \tilde{x}^{k-1}, \widetilde{X}_{t_{k}-t_{k-1}}^{\varepsilon,\left(\tilde{x}^{k-1}, 0\right)}\right)\right], \\
& \vdots \\
\varphi^{k-1}\left(\tilde{x}^{1}\right)= & \lim _{\varepsilon \rightarrow 0} \hat{\mathbb{E}}\left[\varphi^{k-2}\left(\tilde{x}^{1}, \widetilde{X}_{t_{2}-t_{1}}^{\varepsilon,\left(\tilde{x}^{1}, 0\right)}\right)\right] .
\end{aligned}
$$

Remark 3.10 Applying Theorem 3.9 and Kolmogorov's criterion for weak compactness (see Lemma 4.31) to Example 3.4 we can also derive that the slow diffusion process $\widetilde{X}^{\varepsilon, x}$ converges weakly to $\widetilde{X}^{\tilde{x}}$, which is the averaging principle for SDEs introduced by [29]. 


\section{The proof of the main results}

In this section, we shall state the proof of the main results, by making use of nonlinear stochastic calculus and viscosity solution theory, which is different from the linear case. Roughly speaking, we will prove the limit function of $u^{\varepsilon}$ is the unique viscosity solution to the averaged PDE (9). The key point is based on the uniform estimate (7).

First, we establish a uniform a priori estimate of $G$-SDE (11) with two time-scales, which is important for our future discussion. Let $T>0$ be a fixed constant.

Lemma 4.1 Assume that the conditions (H1) and (H2) hold. Then, there exists a constant $C\left(L_{1}, \eta, T\right)$, such that for any $x, x^{\prime} \in \mathbb{R}^{2 n}$ and $t \in[0, T]$,

(i) $\hat{\mathbb{E}}\left[\sup _{0 \leq s \leq T}\left|\tilde{X}_{s}^{\varepsilon, x}-\tilde{X}_{s}^{\varepsilon, x^{\prime}}\right|^{2}\right] \leq C\left(L_{1}, \eta, T\right)\left(\left|\tilde{x}-\tilde{x}^{\prime}\right|^{2}+\varepsilon\left|\bar{x}-\bar{x}^{\prime}\right|^{2}\right)$,

(ii) $\hat{\mathbb{E}}\left[\left|\bar{X}_{t}^{\varepsilon, x}-\bar{X}_{t}^{\varepsilon, x^{\prime}}\right|^{2}\right] \leq C\left(L_{1}, \eta, T\right)\left(\left|\tilde{x}-\tilde{x}^{\prime}\right|^{2}+\left|\bar{x}-\bar{x}^{\prime}\right|^{2}\right)$,

(iii) $\hat{\mathbb{E}}\left[\sup _{0 \leq s \leq T}\left|\widetilde{X}_{s}^{\varepsilon, x}\right|^{2}+\left|\bar{X}_{t}^{\varepsilon, x}\right|^{2}\right] \leq C\left(L_{1}, \eta, T\right)\left(1+|\tilde{x}|^{2}+|\bar{x}|^{2}\right)$.

Proof. Without loss of generality, assume that $\widetilde{h}_{i j}=\bar{h}_{i j}=0, i, j=1, \ldots, d$. The proof is divided into the following two steps.

Step 1 (Assertions (i) and (ii)). Applying G-Itô's formula (Proposition 3.6.3 of [38]) to $e^{\frac{\eta}{\varepsilon} t}\left|\bar{X}_{t}^{\varepsilon, x}-\bar{X}_{t}^{\varepsilon, x^{\prime}}\right|^{2}$ yields that

$$
\begin{aligned}
& e^{\frac{\eta}{\varepsilon} t}\left|\bar{X}_{t}^{\varepsilon, x}-\bar{X}_{t}^{\varepsilon, x^{\prime}}\right|^{2}-\left|\bar{x}-\bar{x}^{\prime}\right|^{2}-M_{t} \\
& =\frac{\eta}{\varepsilon} \int_{0}^{t} e^{\frac{\eta}{\varepsilon} s}\left|\bar{X}_{s}^{\varepsilon, x}-\bar{X}_{s}^{\varepsilon, x^{\prime}}\right|^{2} d s+\frac{2}{\varepsilon} \int_{0}^{t} e^{\frac{\eta}{\varepsilon} s}\left\langle\bar{X}_{s}^{\varepsilon, x}-\bar{X}_{s}^{\varepsilon, x^{\prime}}, \bar{b}\left(\widetilde{X}_{s}^{\varepsilon, x}, \bar{X}_{s}^{\varepsilon, x}\right)-\bar{b}\left(\widetilde{X}_{s}^{\varepsilon, x^{\prime}}, \bar{X}_{s}^{\varepsilon, x^{\prime}}\right)\right\rangle d s \\
& \quad+\frac{1}{\varepsilon} \int_{0}^{t} e^{\frac{\eta}{\varepsilon} s}\left(\bar{\sigma}\left(\widetilde{X}_{s}^{\varepsilon, x}, \bar{X}_{s}^{\varepsilon, x}\right)-\bar{\sigma}\left(\widetilde{X}_{s}^{\varepsilon, x^{\prime}}, \bar{X}_{s}^{\varepsilon, x^{\prime}}\right)\right)^{\top}\left(\bar{\sigma}\left(\widetilde{X}_{s}^{\varepsilon, x}, \bar{X}_{s}^{\varepsilon, x}\right)-\bar{\sigma}\left(\widetilde{X}_{s}^{\varepsilon, x^{\prime}}, \bar{X}_{s}^{\varepsilon, x^{\prime}}\right)\right) d\langle B\rangle_{s},
\end{aligned}
$$

where $M_{t}:=\frac{2}{\sqrt{\varepsilon}} \int_{0}^{t} e^{\frac{\eta}{\varepsilon} s}\left(\bar{X}_{s}^{\varepsilon, x}-\bar{X}_{s}^{\varepsilon, x^{\prime}}\right)\left(\bar{\sigma}\left(\widetilde{X}_{s}^{\varepsilon, x}, \bar{X}_{s}^{\varepsilon, x}\right)-\bar{\sigma}\left(\widetilde{X}_{s}^{\varepsilon, x^{\prime}}, \bar{X}_{s}^{\varepsilon, x^{\prime}}\right)\right) d B_{s}$ is a symmetric $G$-martingale with $M_{0}=0$, i.e., $\hat{\mathbb{E}}\left[M_{t}\right]=-\hat{\mathbb{E}}\left[-M_{t}\right]=0$.

Recalling assumption (H1), we deduce that

$$
\begin{aligned}
& \left\langle\bar{X}_{s}^{\varepsilon, x}-\bar{X}_{s}^{\varepsilon, x^{\prime}}, \bar{b}\left(\widetilde{X}_{s}^{\varepsilon, x}, \bar{X}_{s}^{\varepsilon, x}\right)-\bar{b}\left(\widetilde{X}_{s}^{\varepsilon, x^{\prime}}, \bar{X}_{s}^{\varepsilon, x^{\prime}}\right)\right\rangle \\
& =\left\langle\bar{X}_{s}^{\varepsilon, x}-\bar{X}_{s}^{\varepsilon, x^{\prime}}, \bar{b}\left(\widetilde{X}_{s}^{\varepsilon, x}, \bar{X}_{s}^{\varepsilon, x}\right)-\bar{b}\left(\widetilde{X}_{s}^{\varepsilon, x}, \bar{X}_{s}^{\varepsilon, x^{\prime}}\right)+\bar{b}\left(\widetilde{X}_{s}^{\varepsilon, x}, \bar{X}_{s}^{\varepsilon, x^{\prime}}\right)-\bar{b}\left(\widetilde{X}_{s}^{\varepsilon, x^{\prime}}, \bar{X}_{s}^{\varepsilon, x^{\prime}}\right)\right\rangle \\
& \leq\left\langle\bar{X}_{s}^{\varepsilon, x}-\bar{X}_{s}^{\varepsilon, x^{\prime}}, \bar{b}\left(\widetilde{X}_{s}^{\varepsilon, x}, \bar{X}_{s}^{\varepsilon, x}\right)-\bar{b}\left(\widetilde{X}_{s}^{\varepsilon, x}, \bar{X}_{s}^{\varepsilon, x^{\prime}}\right)\right\rangle+L_{1}\left|\bar{X}_{s}^{\varepsilon, x}-\bar{X}_{s}^{\varepsilon, x^{\prime}}\right|\left|\widetilde{X}_{s}^{\varepsilon, x}-\widetilde{X}_{s}^{\varepsilon, x^{\prime}}\right| .
\end{aligned}
$$

By a similar analysis, we can obtain that

$$
\begin{gathered}
\left(\bar{\sigma}\left(\widetilde{X}_{s}^{\varepsilon, x}, \bar{X}_{s}^{\varepsilon, x}\right)-\bar{\sigma}\left(\widetilde{X}_{s}^{\varepsilon, x^{\prime}}, \bar{X}_{s}^{\varepsilon, x^{\prime}}\right)\right)^{\top}\left(\bar{\sigma}\left(\widetilde{X}_{s}^{\varepsilon, x}, \bar{X}_{s}^{\varepsilon, x}\right)-\bar{\sigma}\left(\widetilde{X}_{s}^{\varepsilon, x^{\prime}}, \bar{X}_{s}^{\varepsilon, x^{\prime}}\right)\right) \\
\leq\left(\bar{\sigma}\left(\widetilde{X}_{s}^{\varepsilon, x}, \bar{X}_{s}^{\varepsilon, x}\right)-\bar{\sigma}\left(\widetilde{X}_{s}^{\varepsilon, x}, \bar{X}_{s}^{\varepsilon, x^{\prime}}\right)\right)^{\top}\left(\bar{\sigma}\left(\widetilde{X}_{s}^{\varepsilon, x}, \bar{X}_{s}^{\varepsilon, x}\right)-\bar{\sigma}\left(\widetilde{X}_{s}^{\varepsilon, x}, \bar{X}_{s}^{\varepsilon, x^{\prime}}\right)\right) \\
+C\left(L_{1}\right)\left(\left|\bar{X}_{s}^{\varepsilon, x}-\bar{X}_{s}^{\varepsilon, x^{\prime}}\right|\left|\widetilde{X}_{s}^{\varepsilon, x}-\widetilde{X}_{s}^{\varepsilon, x^{\prime}}\right|+\left|\widetilde{X}_{s}^{\varepsilon, x}-\widetilde{X}_{s}^{\varepsilon, x^{\prime}}\right|^{2}\right) I_{n} .
\end{gathered}
$$


In view of Corollary 3.5.8 of [38], we have that, for each $\eta \in M_{G}^{1}(0, T ; \mathbb{S}(d))$,

$$
\int_{0}^{t} \eta_{s} d\langle B\rangle_{s}-2 \int_{0}^{t} G\left(\eta_{s}\right) d s \leq 0 .
$$

Then, according to inequality (11), we get that

$$
\begin{aligned}
& e^{\frac{\eta}{\varepsilon} t}\left|\bar{X}_{t}^{\varepsilon, x}-\bar{X}_{t}^{\varepsilon, x^{\prime}}\right|^{2}-\left|\bar{x}-\bar{x}^{\prime}\right|^{2}-M_{t} \\
& \leq \frac{\eta}{\varepsilon} \int_{0}^{t} e^{\frac{\eta}{\varepsilon} s}\left|\bar{X}_{s}^{\varepsilon, x}-\bar{X}_{s}^{\varepsilon, x^{\prime}}\right|^{2} d s+\frac{2}{\varepsilon} \int_{0}^{t} e^{\frac{\eta}{\varepsilon} s}\left\langle\bar{X}_{s}^{\varepsilon, x}-\bar{X}_{s}^{\varepsilon, x^{\prime}}, \bar{b}\left(\widetilde{X}_{s}^{\varepsilon, x}, \bar{X}_{s}^{\varepsilon, x}\right)-\bar{b}\left(\widetilde{X}_{s}^{\varepsilon, x}, \bar{X}_{s}^{\varepsilon, x^{\prime}}\right)\right\rangle d s \\
& \quad+\frac{2}{\varepsilon} \int_{0}^{t} e^{\frac{\eta}{\varepsilon} s} G\left(\left(\bar{\sigma}\left(\widetilde{X}_{s}^{\varepsilon, x}, \bar{X}_{s}^{\varepsilon, x}\right)-\bar{\sigma}\left(\widetilde{X}_{s}^{\varepsilon, x}, \bar{X}_{s}^{\varepsilon, x^{\prime}}\right)\right)^{\top}\left(\bar{\sigma}\left(\widetilde{X}_{s}^{\varepsilon, x}, \bar{X}_{s}^{\varepsilon, x}\right)-\bar{\sigma}\left(\widetilde{X}_{s}^{\varepsilon, x}, \bar{X}_{s}^{\varepsilon, x^{\prime}}\right)\right)\right) d s \\
& \quad+\frac{C\left(L_{1}\right)}{\varepsilon} \int_{0}^{t} e^{\frac{\eta}{\varepsilon} s}\left(\left|\bar{X}_{s}^{\varepsilon, x}-\bar{X}_{s}^{\varepsilon, x^{\prime}}\right|\left|\widetilde{X}_{s}^{\varepsilon, x}-\widetilde{X}_{s}^{\varepsilon, x^{\prime}}\right|+\left|\widetilde{X}_{s}^{\varepsilon, x}-\widetilde{X}_{s}^{\varepsilon, x^{\prime}}\right|^{2}\right) d s,
\end{aligned}
$$

which together with condition (H2) and

$$
\left|\bar{X}_{t}^{\varepsilon, x}-\bar{X}_{t}^{\varepsilon, x^{\prime}}\right|\left|\widetilde{X}_{t}^{\varepsilon, x}-\widetilde{X}_{t}^{\varepsilon, x^{\prime}}\right| \leq \frac{\eta}{C\left(L_{1}\right)}\left|\bar{X}_{t}^{\varepsilon, x}-\bar{X}_{t}^{\varepsilon, x^{\prime}}\right|^{2}+\frac{C\left(L_{1}\right)}{4 \eta}\left|\widetilde{X}_{t}^{\varepsilon, x}-\widetilde{X}_{t}^{\varepsilon, x^{\prime}}\right|^{2}
$$

implies that

$$
e^{\frac{\eta}{\varepsilon} t}\left|\bar{X}_{t}^{\varepsilon, x}-\bar{X}_{t}^{\varepsilon, x^{\prime}}\right|^{2} \leq\left|\bar{x}-\bar{x}^{\prime}\right|^{2}+M_{t}+\frac{C\left(L_{1}, \eta\right)}{\varepsilon} \int_{0}^{t} e^{\frac{\eta}{\varepsilon} s}\left|\widetilde{X}_{s}^{\varepsilon, x}-\widetilde{X}_{s}^{\varepsilon, x^{\prime}}\right|^{2} d s .
$$

Taking $G$-expectation to both sides, we obtain that for each $t \in[0, T]$,

$$
\hat{\mathbb{E}}\left[\left|\bar{X}_{t}^{\varepsilon, x}-\bar{X}_{t}^{\varepsilon, x^{\prime}}\right|^{2}\right] \leq\left|\bar{x}-\bar{x}^{\prime}\right|^{2} e^{-\frac{\eta}{\varepsilon} t}+\frac{C\left(L_{1}, \eta\right)}{\varepsilon} \int_{0}^{t} e^{\frac{\eta}{\varepsilon}(s-t)} \hat{\mathbb{E}}\left[\left|\widetilde{X}_{s}^{\varepsilon, x}-\widetilde{X}_{s}^{\varepsilon, x^{\prime}}\right|^{2}\right] d s
$$

In particular, it holds that

$$
\int_{0}^{t} \hat{\mathbb{E}}\left[\left|\bar{X}_{s}^{\varepsilon, x}-\bar{X}_{s}^{\varepsilon, x^{\prime}}\right|^{2}\right] d s \leq \frac{\varepsilon}{\eta}\left|\bar{x}-\bar{x}^{\prime}\right|^{2}+C\left(L_{1}, \eta\right) \int_{0}^{t} \hat{\mathbb{E}}\left[\sup _{0 \leq r \leq s}\left|\widetilde{X}_{r}^{\varepsilon, x}-\widetilde{X}_{r}^{\varepsilon, x^{\prime}}\right|^{2}\right] d s .
$$

On the other hand, applying Hölder's inequality and BDG's inequality, we conclude that

$$
\begin{aligned}
\hat{\mathbb{E}}\left[\sup _{0 \leq s \leq t}\left|\tilde{X}_{s}^{\varepsilon, x}-\tilde{X}_{s}^{\varepsilon, x^{\prime}}\right|^{2}\right] & \leq 3\left|\tilde{x}-\tilde{x}^{\prime}\right|^{2}+C\left(L_{1}, T\right) \int_{0}^{t} \hat{\mathbb{E}}\left[\left|\bar{X}_{s}^{\varepsilon, x}-\bar{X}_{s}^{\varepsilon, x^{\prime}}\right|^{2}+\left|\tilde{X}_{s}^{\varepsilon, x}-\tilde{X}_{s}^{\varepsilon, x^{\prime}}\right|^{2}\right] d s \\
& \leq C\left(L_{1}, \eta, T\right)\left(\left|\tilde{x}-\tilde{x}^{\prime}\right|^{2}+\varepsilon\left|\bar{x}-\bar{x}^{\prime}\right|^{2}+\int_{0}^{t} \hat{\mathbb{E}}\left[\sup _{0 \leq r \leq s}\left|\widetilde{X}_{r}^{\varepsilon, x}-\tilde{X}_{r}^{\varepsilon, x^{\prime}}\right|^{2}\right] d s\right)
\end{aligned}
$$

where we have used the estimate (13) in the last inequality. It follows from Gronwall's inequality that

$$
\hat{\mathbb{E}}\left[\sup _{0 \leq s \leq T}\left|\widetilde{X}_{s}^{\varepsilon, x}-\widetilde{X}_{s}^{\varepsilon, x^{\prime}}\right|^{2}\right] \leq C\left(L_{1}, \eta, T\right)\left(\left|\tilde{x}-\tilde{x}^{\prime}\right|^{2}+\varepsilon\left|\bar{x}-\bar{x}^{\prime}\right|^{2}\right) .
$$

With the help of inequality (12), we obtain that

$$
\hat{\mathbb{E}}\left[\left|\bar{X}_{t}^{\varepsilon, x}-\bar{X}_{t}^{\varepsilon, x^{\prime}}\right|^{2}\right] \leq C\left(L_{1}, \eta, T\right)\left(\left|\tilde{x}-\tilde{x}^{\prime}\right|^{2}+\left|\bar{x}-\bar{x}^{\prime}\right|^{2}\right), \forall t \in[0, T],
$$


which is the desired result.

Step 2 (Assertion (iii)). Applying G-Itô's formula again, we deduce that

$$
\begin{aligned}
& e^{\frac{\eta}{\varepsilon} t}\left|\bar{X}_{t}^{\varepsilon, x}\right|^{2}-|\bar{x}|^{2}-M_{t}^{\prime}-\frac{\eta}{\varepsilon} \int_{0}^{t} e^{\frac{\eta}{\varepsilon} s}\left|\bar{X}_{s}^{\varepsilon, x}\right|^{2} d s \\
& \leq \frac{2}{\varepsilon} \int_{0}^{t} e^{\frac{\eta}{\varepsilon} s}\left[\left\langle\bar{X}_{s}^{\varepsilon, x}, \bar{b}\left(\widetilde{X}_{s}^{\varepsilon, x}, \bar{X}_{s}^{\varepsilon, x}\right)\right\rangle+G\left(\left(\bar{\sigma}\left(\widetilde{X}_{s}^{\varepsilon, x}, \bar{X}_{s}^{\varepsilon, x}\right)\right)^{\top} \bar{\sigma}\left(\widetilde{X}_{s}^{\varepsilon, x}, \bar{X}_{s}^{\varepsilon, x}\right)\right)\right] d s,
\end{aligned}
$$

where $M_{t}^{\prime}$ is a symmetric $G$-martingale with $M_{0}^{\prime}=0$.

In view of assumptions (H1) and (H2), we get that

$$
\begin{aligned}
& 2\left\langle\bar{X}_{s}^{\varepsilon, x}, \bar{b}\left(\widetilde{X}_{s}^{\varepsilon, x}, \bar{X}_{s}^{\varepsilon, x}\right)\right\rangle+2 G\left(\left(\bar{\sigma}\left(\widetilde{X}_{s}^{\varepsilon, x}, \bar{X}_{s}^{\varepsilon, x}\right)\right)^{\top}\left(\bar{\sigma}\left(\widetilde{X}_{s}^{\varepsilon, x}, \bar{X}_{s}^{\varepsilon, x}\right)\right)\right) \\
& \leq 2\left\langle\bar{X}_{s}^{\varepsilon, x}, \bar{b}\left(\widetilde{X}_{s}^{\varepsilon, x}, \bar{X}_{s}^{\varepsilon, x}\right)-\bar{b}\left(\widetilde{X}_{s}^{\varepsilon, x}, 0\right)\right\rangle+C\left(L_{1}\right)\left(1+\left|\bar{X}_{s}^{\varepsilon, x}\right|+\left|\bar{X}_{s}^{\varepsilon, x}\right|\left|\widetilde{X}_{s}^{\varepsilon, x}\right|+\left|\widetilde{X}_{s}^{\varepsilon, x}\right|^{2}\right) \\
& +2 G\left(\left(\bar{\sigma}\left(\widetilde{X}_{s}^{\varepsilon, x}, \bar{X}_{s}^{\varepsilon, x}\right)-\bar{\sigma}\left(\widetilde{X}_{s}^{\varepsilon, x}, 0\right)\right)^{\top}\left(\bar{\sigma}\left(\widetilde{X}_{s}^{\varepsilon, x}, \bar{X}_{s}^{\varepsilon, x}\right)-\bar{\sigma}\left(\widetilde{X}_{s}^{\varepsilon, x}, 0\right)\right)\right) \\
& \leq-\eta\left|\bar{X}_{s}^{\varepsilon, x}\right|^{2}+C\left(L_{1}, \eta\right)\left(1+\left|\widetilde{X}_{s}^{\varepsilon, x}\right|^{2}\right) \text {. }
\end{aligned}
$$

It follows from inequality (14) that

$$
\hat{\mathbb{E}}\left[\left|\bar{X}_{t}^{\varepsilon, x}\right|^{2}\right] \leq e^{-\frac{\eta}{\varepsilon} t}|\bar{x}|^{2}+\frac{C\left(L_{1}, \eta\right)}{\varepsilon} \int_{0}^{t} e^{\frac{\eta}{\varepsilon}(s-t)}\left(1+\hat{\mathbb{E}}\left[\left|\widetilde{X}_{s}^{\varepsilon, x}\right|^{2}\right]\right) d s, \forall t \in[0, T] .
$$

On the other hand, using inequality (15) and by a similar analysis as step 1, we obtain that

$$
\begin{aligned}
\hat{\mathbb{E}}\left[\sup _{0 \leq s \leq t}\left|\widetilde{X}_{s}^{\varepsilon, x}\right|^{2}\right] & \leq 3|\tilde{x}|^{2}+C\left(L_{1}, T\right) \int_{0}^{t} \hat{\mathbb{E}}\left[1+\left|\bar{X}_{s}^{\varepsilon, x}\right|^{2}+\left|\widetilde{X}_{s}^{\varepsilon, x}\right|^{2}\right] d s \\
& \leq C\left(L_{1}, \eta, T\right)\left(1+|\tilde{x}|^{2}+\varepsilon|\bar{x}|^{2}+\int_{0}^{t} \hat{\mathbb{E}}\left[\sup _{0 \leq r \leq s}\left|\widetilde{X}_{r}^{\varepsilon, x}\right|^{2}\right] d s\right) .
\end{aligned}
$$

Consequently, it holds that

$$
\hat{\mathbb{E}}\left[\sup _{0 \leq s \leq T}\left|\widetilde{X}_{s}^{\varepsilon, x}\right|^{2}\right]+\sup _{0 \leq t \leq T} \hat{\mathbb{E}}\left[\left|\bar{X}_{t}^{\varepsilon, x}\right|^{2}\right] \leq C\left(L_{1}, \eta, T\right)\left(1+|\tilde{x}|^{2}+|\bar{x}|^{2}\right) .
$$

The proof is complete.

Then, we have the following asymptotic properties of $u^{\varepsilon}$.

Lemma 4.2 Let $\varphi$ be in $C_{b . l i p}\left(\mathbb{R}^{n}\right)$. Suppose assumptions (H1) and (H2) are satisfied. Then, there exist a sequence $\varepsilon_{m} \downarrow 0, m \geq 1$ and a function $\widetilde{u}^{*} \in C_{b}\left([0, T] \times \mathbb{R}^{n}\right)$, such that for each $s, t \in[0, T]$ and $x=(\tilde{x}, \bar{x}) \in \mathbb{R}^{2 n}$,

(i) $\lim _{m \rightarrow \infty} u^{\varepsilon_{m}}(t, \tilde{x}, \bar{x})=\widetilde{u}^{*}(t, \tilde{x})$,

(ii) $\lim _{m \rightarrow \infty} \hat{\mathbb{E}}\left[\left|u^{\varepsilon_{m}}\left(s, \widetilde{X}_{t}^{\varepsilon_{m}, x}, \bar{X}_{t}^{\varepsilon_{m}, x}\right)-\widetilde{u}^{*}\left(s, \widetilde{X}_{t}^{\varepsilon_{m}, x}\right)\right|\right]=0$. 
Proof. From Lemma 4.1, we have that, for any $s, t \in[0, T]$ and $x, x^{\prime} \in \mathbb{R}^{2 n}$,

$$
\begin{aligned}
\hat{\mathbb{E}}\left[\left|\widetilde{X}_{t}^{\varepsilon, x}-\widetilde{X}_{s}^{\varepsilon, x^{\prime}}\right|^{2}\right] & \leq 2 \hat{\mathbb{E}}\left[\left|\widetilde{X}_{t}^{\varepsilon, x}-\widetilde{X}_{s}^{\varepsilon, x}\right|^{2}\right]+2 \hat{\mathbb{E}}\left[\left|\widetilde{X}_{s}^{\varepsilon, x}-\widetilde{X}_{s}^{\varepsilon, x^{\prime}}\right|^{2}\right] \\
& \leq C\left(L_{1}, \eta, T\right)\left(\left(1+|x|^{2}\right)|t-s|+\left|\tilde{x}-\tilde{x}^{\prime}\right|^{2}+\varepsilon\left|\bar{x}-\bar{x}^{\prime}\right|^{2}\right) .
\end{aligned}
$$

It follows from the definition of $u^{\varepsilon}$ that $\left|u^{\varepsilon}(t, \tilde{x}, \bar{x})\right| \leq C(\varphi)$ and

$$
\begin{aligned}
\left|u^{\varepsilon}(t, \tilde{x}, \bar{x})-u^{\varepsilon}\left(s, \tilde{x}^{\prime}, \bar{x}^{\prime}\right)\right| & \leq \hat{\mathbb{E}}\left[\left|\varphi\left(\widetilde{X}_{t}^{\varepsilon, x}\right)-\varphi\left(\widetilde{X}_{s}^{\varepsilon, x^{\prime}}\right)\right|\right] \leq C(\varphi) \hat{\mathbb{E}}\left[\left|\widetilde{X}_{t}^{\varepsilon, x}-\widetilde{X}_{s}^{\varepsilon, x^{\prime}}\right|\right] \\
& \leq C\left(L_{1}, \eta, T, \varphi\right)\left((1+|x|) \sqrt{|t-s|}+\left|\tilde{x}-\tilde{x}^{\prime}\right|+\sqrt{\varepsilon}\left|\bar{x}-\bar{x}^{\prime}\right|\right) .
\end{aligned}
$$

Thus, by the Arzelà-Ascoli theorem, we can find a sequence $\varepsilon_{m} \downarrow 0$, such that $u^{\varepsilon_{m}}(t, \tilde{x}, \bar{x})$ is a Cauchy sequence for any $(t, \tilde{x}, \bar{x}) \in[0, T] \times \mathbb{R}^{2 n}$. Denote $\widetilde{u}^{*}(t, \tilde{x}, \bar{x}):=\lim _{m \rightarrow \infty} u^{\varepsilon_{m}}(t, \tilde{x}, \bar{x})$. It is obvious that

$$
\left|\widetilde{u}^{*}(t, \tilde{x}, \bar{x})-\widetilde{u}^{*}\left(s, \tilde{x}^{\prime}, \bar{x}^{\prime}\right)\right| \leq C\left(L_{1}, \eta, T, \varphi\right)\left((1+|x|) \sqrt{|t-s|}+\left|\tilde{x}-\tilde{x}^{\prime}\right|\right), \forall \bar{x}, \bar{x}^{\prime} \in \mathbb{R}^{n},
$$

which indicates that $\widetilde{u}^{*}$ is independent of the argument $\bar{x}$.

Next, we will prove Assertion (ii). For each $N>0$, we get that

$$
\begin{aligned}
& \hat{\mathbb{E}}\left[\left|u^{\varepsilon_{m}}\left(s, \widetilde{X}_{t}^{\varepsilon_{m}, x}, \bar{X}_{t}^{\varepsilon_{m}, x}\right)-\widetilde{u}^{*}\left(s, \widetilde{X}_{t}^{\varepsilon_{m}, x}\right)\right|\right] \\
& \leq \hat{\mathbb{E}}\left[\left|u^{\varepsilon_{m}}\left(s, \widetilde{X}_{t}^{\varepsilon_{m}, x}, \bar{X}_{t}^{\varepsilon_{m}, x}\right)-\widetilde{u}^{*}\left(s, \widetilde{X}_{t}^{\varepsilon_{m}, x}\right)\right| I_{\left\{\left|\widetilde{X}_{t}^{\varepsilon_{m}, x}\right| \leq N\right\}} I_{\left\{\left|\bar{X}_{t}^{\varepsilon_{m}, x}\right| \leq N\right\}}\right] \\
& +C(\varphi) \hat{\mathbb{E}}\left[I_{\left\{\left|\tilde{X}_{t}^{\varepsilon m, x}\right| \geq N\right\}}+I_{\left.\left\{\left|\bar{X}_{t}^{\varepsilon_{m}, x}\right| \geq N\right\}\right]}\right] \\
& \leq \sup _{s \in[0, T],|\tilde{x}|,|\bar{x}| \leq N}\left|u^{\varepsilon_{m}}(s, \tilde{x}, \bar{x})-\widetilde{u}^{*}(s, \tilde{x})\right|+\frac{C(\varphi)}{N} \hat{\mathbb{E}}\left[\left|\widetilde{X}_{t}^{\varepsilon_{m}, x}\right|+\left|\bar{X}_{t}^{\varepsilon_{m}, x}\right|\right] .
\end{aligned}
$$

Note that $u^{\varepsilon_{m}}$ converges uniformly to $\widetilde{u}^{*}$ on every compact subset of $[0, T] \times \mathbb{R}^{2 n}$. Thus, with the help Assertion (iii) of Lemma 4.1 we conclude that

$$
\limsup _{m \rightarrow \infty} \hat{\mathbb{E}}\left[\left|u^{\varepsilon_{m}}\left(s, \widetilde{X}_{t}^{\varepsilon_{m}, x}, \bar{X}_{t}^{\varepsilon_{m}, x}\right)-\widetilde{u}^{*}\left(s, \widetilde{X}_{t}^{\varepsilon_{m}, x}\right)\right|\right] \leq \frac{C\left(L_{1}, \eta, T, \varphi\right)}{N}(1+|\tilde{x}|+|\bar{x}|), \forall N>0 .
$$

Sending $N \rightarrow \infty$ yields the desired result.

Next, we show that the function $\widetilde{u}^{*}$ constructed above is the viscosity solution to PDE (9). For this purpose, we need the following two lemmas.

Lemma 4.3 Suppose (H1) and (H3) hold. Then, for any $p \geq 2$ and $t, s \in[0, T]$,

$$
\begin{aligned}
& \hat{\mathbb{E}}\left[\sup _{0 \leq s \leq T}\left|\widetilde{X}_{s}^{\varepsilon, x}\right|^{p}\right] \leq C\left(L_{1}, L_{2}, p, T\right)\left(1+|\tilde{x}|^{p}\right), \\
& \hat{\mathbb{E}}\left[\left|\widetilde{X}_{t}^{\varepsilon, x}-\widetilde{X}_{s}^{\varepsilon, x}\right|^{p}\right] \leq C\left(L_{1}, L_{2}, p, T\right)\left(1+|\tilde{x}|^{p}\right)|t-s|^{\frac{p}{2}} .
\end{aligned}
$$

Proof. The proof is immediate from BDG's inequality and Gronwall's inequality.

Lemma 4.4 Suppose (H1) holds. Then, for each $\rho \in L_{G}^{1}(\Omega)$ and element $\Gamma \in \mathbb{S}(n)$, it holds that

$$
\hat{\mathbb{E}}\left[\rho+\left\langle\Gamma \int_{0}^{t} \widetilde{\sigma}\left(X_{s}^{\varepsilon, x}\right) d B_{s}, \int_{0}^{t} \widetilde{\sigma}\left(X_{s}^{\varepsilon, x}\right) d B_{s}\right\rangle-\int_{0}^{t} \widetilde{\sigma}^{\Gamma}\left(X_{s}^{\varepsilon, x}\right) d\langle B\rangle_{s}\right]=\hat{\mathbb{E}}[\rho], \forall t>0,
$$

where $\widetilde{\sigma}^{\Gamma}$ is given by Lemma 3.2 . 
Proof. Suppose that $\left\{M_{t}\right\}_{t>0}$ is a symmetric $G$-martingale, i.e., $-\hat{\mathbb{E}}\left[-M_{t}\right]=\hat{\mathbb{E}}\left[M_{t}\right]$. Then, using the property of $G$-expectation (Proposition 1.3.7 of [38]), we get that

$$
\hat{\mathbb{E}}\left[\rho+M_{t}\right]=\hat{\mathbb{E}}[\rho] .
$$

On the other hand, applying $G$-Itô's formula and recalling the definition of $\widetilde{\sigma}^{\Gamma}$, we can get that,

$$
\left\langle\Gamma \int_{0}^{t} \widetilde{\sigma}\left(X_{s}^{\varepsilon, x}\right) d B_{s}, \int_{0}^{t} \widetilde{\sigma}\left(X_{s}^{\varepsilon, x}\right) d B_{s}\right\rangle-\int_{0}^{t} \widetilde{\sigma}^{\Gamma}\left(X_{s}^{\varepsilon, x}\right) d\langle B\rangle_{s}
$$

is a symmetric $G$-martingale. It follows from equation (16) that the desired result holds.

Lemma 4.5 Assume all the conditions of Lemma 4.2 are satisfied. Furthermore, suppose assumption (H3) holds. Then, $\widetilde{u}^{*}$ is the unique viscosity solution to PDE (91).

Proof. The uniqueness can be obtained by applying Lemma B.1 in appendix B. It suffices to prove that $\widetilde{u}^{*}$ is a viscosity subsolution, since the other case can be proved in a similar fashion. Without loss of generality, assume that $\widetilde{h}_{i j}, \bar{h}_{i j}=0, i, j=1, \ldots, d$.

Note that $\widetilde{u}^{*}$ is a bounded function. Then, assume that the test function $\psi \in C_{b}^{3}\left([0, T] \times \mathbb{R}^{n}\right)$ satisfies that $\psi \geq \widetilde{u}^{*}$ and $\psi(t, \tilde{x})=\widetilde{u}^{*}(t, \tilde{x})$ for some point $(t, \tilde{x}) \in(0, T) \times \mathbb{R}^{n}$, where $C_{b}^{3}\left([0, T] \times \mathbb{R}^{n}\right)$ is the space of the bounded real-valued functions that are continuously differentiable up to the third order and whose derivatives of order from 1 to 3 are bounded. We need to prove that

$$
H(t, \tilde{x}, \psi):=\partial_{t} \psi(t, \tilde{x})-\widetilde{G}\left(\tilde{x}, D \psi(t, \tilde{x}), D^{2} \psi(t, \tilde{x})\right) \leq 0 .
$$

The proof is divided into the following four steps.

Step 1 (Dynamic programming principle). Use the same notations as Lemma 4.2 Recalling equation (4), we obtain that, for each $\delta \in(0,1)$ and for any $\bar{x} \in \mathbb{R}^{n}$

$$
u^{\varepsilon_{m}}(t, \tilde{x}, \bar{x})=\hat{\mathbb{E}}\left[u^{\varepsilon_{m}}\left(t-\delta, \widetilde{X}_{\delta}^{\varepsilon_{m}, x}, \bar{X}_{\delta}^{\varepsilon_{m}, x}\right)\right],
$$

which together with Assertion (ii) of Lemma 4.2 implies that

$$
\widetilde{u}^{*}(t, \tilde{x})=\lim _{m \rightarrow \infty} \hat{\mathbb{E}}\left[\widetilde{u}^{*}\left(t-\delta, \widetilde{X}_{\delta}^{\varepsilon_{m}, x}\right)\right] .
$$

It follows that

$$
\psi(t, \tilde{x}) \leq \limsup _{m \rightarrow \infty} \hat{\mathbb{E}}\left[\psi\left(t-\delta, \widetilde{X}_{\delta}^{\varepsilon_{m}, x}\right)\right] .
$$

Step 2 (The subsolution property). For each $m \geq 1$, define

$$
\xi^{1, m}=\int_{0}^{\delta} \widetilde{b}\left(\widetilde{X}_{s}^{\varepsilon_{m}, x}, \bar{X}_{s}^{\varepsilon_{m}, x}\right) d s \text { and } \xi^{2, m}=\int_{0}^{\delta} \widetilde{\sigma}\left(\widetilde{X}_{s}^{\varepsilon_{m}, x}, \bar{X}_{s}^{\varepsilon_{m}, x}\right) d B_{s} .
$$

Note that

$$
\psi\left(t-\delta, \widetilde{X}_{\delta}^{\varepsilon_{m}, x}\right)-\psi(t, \tilde{x})=\psi\left(t-\delta, \widetilde{X}_{\delta}^{\varepsilon_{m}, x}\right)-\psi\left(t, \widetilde{X}_{\delta}^{\varepsilon_{m}, x}\right)+\psi\left(t, \widetilde{X}_{\delta}^{\varepsilon_{m}, x}\right)-\psi(t, \tilde{x}) .
$$

Then, applying Taylor's expansion yields that,

$$
\begin{aligned}
& \psi\left(t-\delta, \widetilde{X}_{\delta}^{\varepsilon_{m}, x}\right)-\psi\left(t, \widetilde{X}_{\delta}^{\varepsilon_{m}, x}\right)=-\partial_{t} \psi(t, \tilde{x}) \delta+\epsilon^{1, m} \\
& \psi\left(t, \widetilde{X}_{\delta}^{\varepsilon_{m}, x}\right)-\psi(t, \tilde{x})=\left\langle D \psi(t, \tilde{x}),\left(\xi^{1, m}+\xi^{2, m}\right)\right\rangle+\frac{1}{2}\left\langle D^{2} \psi(t, \tilde{x}) \xi^{2, m}, \xi^{2, m}\right\rangle+\epsilon^{2, m},
\end{aligned}
$$


with

$$
\begin{aligned}
\epsilon^{1, m}= & \delta \int_{0}^{1}\left[-\partial_{t} \psi\left(t-\alpha \delta, \widetilde{X}_{\delta}^{\varepsilon_{m}, x}\right)+\partial_{t} \psi(t, \tilde{x})\right] d \alpha \\
\epsilon^{2, m}= & \int_{0}^{1}\left\langle D \psi\left(t, \tilde{x}+\xi^{2, m}+\alpha \xi^{1, m}\right)-D \psi(t, \tilde{x}), \xi^{1, m}\right\rangle d \alpha \\
& \quad+\int_{0}^{1} \int_{0}^{1}\left\langle\left(D^{2} \psi\left(t, \tilde{x}+\alpha \beta \xi^{2, m}\right)-D^{2} \psi(t, \tilde{x})\right) \xi^{2, m}, \xi^{2, m}\right\rangle \alpha d \beta d \alpha .
\end{aligned}
$$

Denote $J^{m}$ by

$$
J^{m}:=-\partial_{t} \psi(t, \tilde{x}) \delta+\left\langle D \psi(t, \tilde{x}), \xi^{1, m}\right\rangle+\gamma^{m} \text { and } \gamma^{m}:=\frac{1}{2} \int_{0}^{\delta} \widetilde{\sigma}^{D^{2} \psi(t, \tilde{x})}\left(\widetilde{X}_{s}^{\varepsilon_{m}, x}, \bar{X}_{s}^{\varepsilon_{m}, x}\right) d\langle B\rangle_{s} .
$$

In view of the equation (19), we deduce that

$$
\psi\left(t-\delta, \widetilde{X}_{\delta}^{\varepsilon_{m}, x}\right)-\psi(t, \tilde{x})=J^{m}+\frac{1}{2}\left\langle D^{2} \psi(t, \tilde{x}) \xi^{2, m}, \xi^{2, m}\right\rangle-\gamma^{m}+\left\langle D \psi(t, \tilde{x}), \xi^{2, m}\right\rangle+\epsilon^{1, m}+\epsilon^{2, m} .
$$

Note that $\left\langle D \psi(t, \tilde{x}), \xi^{2, m}\right\rangle$ has no mean uncertainty. Thus, with the help of Lemma 4.4 we obtain that

$$
\left|\hat{\mathbb{E}}\left[\psi\left(t-\delta, \widetilde{X}_{\delta}^{\varepsilon_{m}, x}\right)\right]-\psi(t, \tilde{x})-\hat{\mathbb{E}}\left[J^{m}\right]\right|=\left|\hat{\mathbb{E}}\left[J^{m}+\epsilon^{1, m}+\epsilon^{2, m}\right]-\hat{\mathbb{E}}\left[J^{m}\right]\right| \leq \hat{\mathbb{E}}\left[\left|\epsilon^{1, m}\right|+\left|\epsilon^{2, m}\right|\right] .
$$

Note that $\delta<1$. Recalling assumption (H3) and Lemma 4.3 we get that

$$
\hat{\mathbb{E}}\left[\left|\xi^{1, m}\right|^{2}\right] \leq C\left(L_{1}, L_{2}\right)\left(1+|\tilde{x}|^{2}\right)|\delta|^{2} \text { and } \hat{\mathbb{E}}\left[\left|\xi^{2, m}\right|^{3}\right] \leq C\left(L_{1}, L_{2}\right)\left(1+|\tilde{x}|^{3}\right)|\delta|^{\frac{3}{2}} .
$$

Then, from the definition of $\epsilon^{i, m}, i=1,2$, we conclude that

$$
\hat{\mathbb{E}}\left[\left|\epsilon^{1, m}\right|+\left|\epsilon^{2, m}\right|\right] \leq C\left(L_{1}, L_{2}, \psi\right)\left(1+|\tilde{x}|^{3}\right)|\delta|^{\frac{3}{2}},
$$

which indicates that

$$
\left|\hat{\mathbb{E}}\left[\psi\left(t-\delta, \widetilde{X}_{\delta}^{\varepsilon_{m}, x}\right)\right]-\psi(t, \tilde{x})-\hat{\mathbb{E}}\left[J^{m}\right]\right| \leq C\left(L_{1}, L_{2}, \psi\right)\left(1+|\tilde{x}|^{3}\right)|\delta|^{\frac{3}{2}}
$$

We claim that

$$
\left|\hat{\mathbb{E}}\left[J^{m}\right]+H(t, \tilde{x}, \psi) \delta\right| \leq C\left(L_{1}, L_{2}, \eta, \psi\right)\left(1+|x|^{3}\right)\left(\frac{\varepsilon_{m}}{\delta_{m}}+\sqrt{\delta_{m}}+\sqrt{\delta}+\sqrt{\rho_{m}}\right) \delta,
$$

whose proof will be given in step 4 . Here the constants $\delta_{m}$ and $\rho_{m}$ will be given in step 3 .

From the inequalities (20) and (21), we derive that

$$
\left|\hat{\mathbb{E}}\left[\psi\left(t-\delta, \widetilde{X}_{\delta}^{\varepsilon_{m}, x}\right)\right]-\psi(t, \tilde{x})+H(t, \tilde{x}, \psi) \delta\right| \leq C\left(L_{1}, L_{2}, \eta, \psi\right)\left(1+|x|^{3}\right)\left(\frac{\varepsilon_{m}}{\delta_{m}}+\sqrt{\delta_{m}}+\sqrt{\delta}+\sqrt{\rho_{m}}\right) \delta,
$$

which implies that

$\frac{1}{\delta}\left(\hat{\mathbb{E}}\left[\psi\left(t-\delta, \widetilde{X}_{\delta}^{\varepsilon_{m}, x}\right)\right]-\psi(t, \tilde{x})\right) \leq-H(t, \tilde{x}, \psi)+C\left(L_{1}, L_{2}, \eta, \psi\right)\left(1+|x|^{3}\right)\left(\frac{\varepsilon_{m}}{\delta_{m}}+\sqrt{\delta_{m}}+\sqrt{\delta}+\sqrt{\rho_{m}}\right)$.

Consequently, we put the above inequality into the equation (18), and obtain that, for each $\delta \in(0,1)$,

$$
0 \leq \limsup _{m \rightarrow \infty} \frac{1}{\delta}\left(\hat{\mathbb{E}}\left[\psi\left(t-\delta, \widetilde{X}_{\delta}^{\varepsilon_{m}, x}\right)\right]-\psi(t, \tilde{x})\right) \leq-H(t, \tilde{x}, \psi)+C\left(L_{1}, L_{2}, \eta, \psi\right)\left(1+|x|^{3}\right) \sqrt{\delta},
$$


where we have used the fact that $\delta_{m}, \frac{\varepsilon_{m}}{\delta_{m}}, \rho_{m}$ converge to 0 as $m \rightarrow \infty$. Sending $\delta \rightarrow 0$ yields that $H(t, \tilde{x}, \psi) \leq 0$, which is the desired result.

Step 3 (Khasminskii's discretization). In order to prove the inequality (21), we shall introduce Khasminskii's discretization for the fast component $\bar{X}^{\varepsilon, x}$. For each positive integer $m$, we define

$$
\delta_{m}:=\varepsilon_{m} \sqrt[4]{\ln \varepsilon_{m}^{-1}} .
$$

Note that $\delta_{m}$ converges to 0 as $m \rightarrow \infty$. Then, we split the time interval $[0, \delta]$ in length $\delta_{m}$ for large enough $m$. Next, consider the following auxiliary stochastic process, for any $s \in\left[l \delta_{m},(l+1) \delta_{m} \wedge \delta\right)$, $l=0, \ldots, N^{m}$ with $N^{m}:=\left[\frac{\delta}{\delta_{m}}\right]$,

$$
\bar{X}_{s}^{D, \varepsilon_{m}, x}=\bar{X}_{l \delta_{m}}^{\varepsilon_{m}, x}+\int_{l \delta_{m}}^{s} \frac{\bar{b}\left(\widetilde{X}_{l \delta_{m}}^{\varepsilon_{m}, x}, \bar{X}_{r}^{D, \varepsilon_{m}, x}\right)}{\varepsilon_{m}} d r+\int_{l \delta_{m}}^{s} \frac{\bar{\sigma}\left(\widetilde{X}_{l \delta_{m}}^{\varepsilon_{m}, x}, \bar{X}_{r}^{D, \varepsilon_{m}, x}\right)}{\sqrt{\varepsilon_{m}}} d B_{r},
$$

which is well-posed in light of the assumption (H1).

By Lemma 4.3 and a standard calculus for $G$-SDEs, we could get that, for any $s \in\left[l \delta_{m},(l+1) \delta_{m} \wedge \delta\right)$,

$$
\begin{aligned}
& \hat{\mathbb{E}}\left[\left|\bar{X}_{s}^{\varepsilon_{m}, x}-\bar{X}_{s}^{D, \varepsilon_{m}, x}\right|^{2}\right] \\
& \leq C\left(L_{1}\right)\left(\frac{\delta_{m}}{\left|\varepsilon_{m}\right|^{2}}+\frac{1}{\varepsilon_{m}}\right) \int_{l \delta_{m}}^{s}\left(\hat{\mathbb{E}}\left[\left|\widetilde{X}_{r}^{\varepsilon_{m}, x}-\widetilde{X}_{l \delta_{m}}^{\varepsilon_{m}, x}\right|^{2}\right]+\hat{\mathbb{E}}\left[\left|\bar{X}_{r}^{\varepsilon_{m}, x}-\bar{X}_{r}^{D, \varepsilon_{m}, x}\right|^{2}\right]\right) d r \\
& \leq C\left(L_{1}, L_{2}\right)\left(\frac{\delta_{m}}{\left|\varepsilon_{m}\right|^{2}}+\frac{1}{\varepsilon_{m}}\right)\left(\left(1+|\tilde{x}|^{2}\right)\left|\delta_{m}\right|^{2}+\int_{l \delta_{m}}^{s} \hat{\mathbb{E}}\left[\left|\bar{X}_{r}^{\varepsilon_{m}, x}-\bar{X}_{r}^{D, \varepsilon_{m}, x}\right|^{2}\right] d r\right),
\end{aligned}
$$

which together with Gronwall's inequality implies that,

$$
\hat{\mathbb{E}}\left[\left|\bar{X}_{s}^{\varepsilon_{m}, x}-\bar{X}_{s}^{D, \varepsilon_{m}, x}\right|^{2}\right] \leq\left(1+|\tilde{x}|^{2}\right) \rho_{m}
$$

with

$$
\rho_{m}:=C\left(L_{1}, L_{2}\right)\left(\frac{\delta_{m}}{\left|\varepsilon_{m}\right|^{2}}+\frac{1}{\varepsilon_{m}}\right)\left|\delta_{m}\right|^{2} \exp \left(C\left(L_{1}, L_{2}\right)\left(\frac{\delta_{m}}{\left|\varepsilon_{m}\right|^{2}}+\frac{1}{\varepsilon_{m}}\right) \delta_{m}\right) .
$$

Recalling the choice of $\delta_{m}$, one can easily check that $\rho_{m}$ converges to 0 as $m \rightarrow \infty$.

Step 4 (The proof of the inequality (21)). For each $l=0, \ldots, N^{m}$, set

$$
\begin{aligned}
& \xi^{1, m, l}=\int_{l \delta_{m}}^{(l+1) \delta_{m} \wedge \delta} \widetilde{b}\left(\widetilde{X}_{s}^{\varepsilon_{m}, x}, \bar{X}_{s}^{\varepsilon_{m}, x}\right) d s, \quad \xi^{D, 1, m, l}=\int_{l \delta_{m}}^{(l+1) \delta_{m} \wedge \delta} \widetilde{b}\left(\widetilde{X}_{l \delta_{m}}^{\varepsilon_{m}, x}, \bar{X}_{s}^{D, \varepsilon_{m}, x}\right) d s, \\
& \gamma^{D, m, l}=\frac{1}{2} \sum_{i, j=1}^{d} \int_{l \delta_{m}}^{(l+1) \delta_{m} \wedge \delta} \widetilde{\sigma}_{i j}^{D^{2} \psi(t, \tilde{x})}\left(\widetilde{X}_{l \delta_{m}}^{\varepsilon_{m}, x}, \bar{X}_{s}^{D, \varepsilon_{m}, x}\right) d\left\langle B^{i}, B^{j}\right\rangle_{s},
\end{aligned}
$$

and

$$
J^{D, m, l}:=-\partial_{t} \psi(t, \tilde{x})\left((l+1) \delta_{m} \wedge \delta-l \delta_{m}\right)+\left\langle D \psi(t, \tilde{x}), \xi^{D, 1, m, l}\right\rangle+\gamma^{D, m, l} .
$$

From Lemma 4.3 and the equation (22), we get that

$$
\begin{aligned}
& \hat{\mathbb{E}}\left[\left|\left\langle D \psi(t, \tilde{x}), \xi^{1, m}\right\rangle-\sum_{l=0}^{N^{m}}\left\langle D \psi(t, \tilde{x}), \xi^{D, 1, m, l}\right\rangle\right|\right] \leq \sum_{l=0}^{N^{m}} C(\psi) \hat{\mathbb{E}}\left[\left|\xi^{1, m, l}-\xi^{D, 1, m, l}\right|\right] \\
& \leq \sum_{l=0}^{N^{m}} C\left(L_{1}, \psi\right) \int_{l \delta_{m}}^{(l+1) \delta_{m} \wedge \delta} \hat{\mathbb{E}}\left[\left|\widetilde{X}_{s}^{\varepsilon_{m}, x}-\widetilde{X}_{l \delta_{m}}^{\varepsilon_{m}, x}\right|+\left|\bar{X}_{s}^{\varepsilon_{m}, x}-\bar{X}_{s}^{D, \varepsilon_{m}, x}\right|\right] d s \\
& \leq \sum_{l=0}^{N^{m}} C\left(L_{1}, L_{2}, \psi\right)(1+|\tilde{x}|)\left(\sqrt{\delta_{m}}+\sqrt{\rho_{m}}\right) \delta_{m} \leq C\left(L_{1}, L_{2}, \psi\right)(1+|\tilde{x}|)\left(\sqrt{\delta_{m}}+\sqrt{\rho_{m}}\right) \delta,
\end{aligned}
$$


where we have used the fact that $N^{m} \delta_{m} \leq \delta \leq\left(N^{m}+1\right) \delta_{m}$ in the last inequality. By a similar way, we could obtain that

$$
\hat{\mathbb{E}}\left[\left|\gamma^{m}-\sum_{l=0}^{N^{m}} \gamma^{D, m, l}\right|\right] \leq C\left(L_{1}, L_{2}, \psi\right)\left(1+|\tilde{x}|^{2}\right)\left(\sqrt{\delta_{m}}+\sqrt{\rho_{m}}\right) \delta .
$$

Thus, it follows from the definition of $J^{m}$ that

$$
\begin{aligned}
\hat{\mathbb{E}}\left[\left|J^{m}-\sum_{l=0}^{N^{m}} J^{D, m, l}\right|\right] & =\hat{\mathbb{E}}\left[\left|\left\langle D \psi(t, \tilde{x}), \xi^{1, m}\right\rangle+\gamma^{m}-\sum_{l=0}^{N^{m}}\left\langle D \psi(t, \tilde{x}), \xi^{D, 1, m, l}\right\rangle-\sum_{l=0}^{N^{m}} \gamma^{D, m, l}\right|\right] \\
& \leq C\left(L_{1}, L_{2}, \psi\right)\left(1+|\tilde{x}|^{2}\right)\left(\sqrt{\delta_{m}}+\sqrt{\rho_{m}}\right) \delta .
\end{aligned}
$$

Now, with the help of Assertion (iv) of Lemma 3.3 and Lemma 4.3, we have that,

$$
\begin{aligned}
& \hat{\mathbb{E}}\left[\left|\widetilde{G}\left(\widetilde{X}_{l \delta_{m}}^{\varepsilon_{m}, x}, D \psi(t, \tilde{x}), D^{2} \psi(t, \tilde{x})\right)-\widetilde{G}\left(\tilde{x}, D \psi(t, \tilde{x}), D^{2} \psi(t, \tilde{x})\right)\right|\right] \\
& \leq C\left(L_{1}, L_{2}, \eta\right)\left(|D \psi(t, \tilde{x})|+\left|D^{2} \psi(t, \tilde{x})\right|\right) \hat{\mathbb{E}}\left[\left(1+\left|\widetilde{X}_{l \delta_{m}, x}^{\varepsilon_{m}, x}\right|^{2}+|\tilde{x}|^{2}\right)\left|\widetilde{X}_{l \delta_{m}}^{\varepsilon_{m}, x}-\tilde{x}\right|\right] \\
& \leq C\left(L_{1}, L_{2}, \eta, \psi\right)\left(\hat{\mathbb{E}}\left[\left(1+\left|\widetilde{X}_{l \delta_{m}}^{\varepsilon_{m}, x}\right|^{2}+|\tilde{x}|^{2}\right)^{2}\right] \hat{\mathbb{E}}\left[\left|\widetilde{X}_{l \delta_{m}}^{\varepsilon_{m}, x}-\tilde{x}\right|^{2}\right]\right)^{\frac{1}{2}} \leq C\left(L_{1}, L_{2}, \eta, \psi\right)\left(1+|\tilde{x}|^{3}\right) \sqrt{\delta} .
\end{aligned}
$$

On the other hand, applying Lemma 4.1 and Lemma 4.7yields that,

$$
\begin{aligned}
& \hat{\mathbb{E}}\left[\left|\hat{\mathbb{E}}_{l \delta_{m}}\left[\left\langle D \psi(t, \tilde{x}), \xi^{D, 1, m, l}\right\rangle+\gamma^{D, m, l}\right]-\widetilde{G}\left(\widetilde{X}_{l \delta_{m}}^{\varepsilon_{m}, x}, D \psi(t, \tilde{x}), D^{2} \psi(t, \tilde{x})\right)\left((l+1) \delta_{m} \wedge \delta-l \delta_{m}\right)\right|\right] \\
& \leq C\left(L_{1}, L_{2}, \eta, \psi\right) \hat{\mathbb{E}}\left[\left(1+\left|\widetilde{X}_{l \delta_{m}}^{\varepsilon_{m}, x}\right|^{2}+\left|\bar{X}_{l \delta_{m}}^{\varepsilon_{m}, x}\right|^{2}\right)\right] \varepsilon_{m} \leq C\left(L_{1}, L_{2}, \eta, \psi\right)\left(1+|x|^{2}\right) \varepsilon_{m} .
\end{aligned}
$$

Then, by the definition of $H$ (see inequality (17)) and the above two inequalities, we derive that, for each $l=0,1, \ldots, N^{m}$,

$$
\begin{aligned}
& \hat{\mathbb{E}}\left[\left|\hat{\mathbb{E}}_{l \delta_{m}}\left[J^{D, m, l}\right]+H(t, \tilde{x}, \psi)\left((l+1) \delta_{m} \wedge \delta-l \delta_{m}\right)\right|\right] \\
& =\hat{\mathbb{E}}\left[\left|\hat{\mathbb{E}}_{l \delta_{m}}\left[\left\langle D \psi(t, \tilde{x}), \xi^{D, 1, m, l}\right\rangle+\gamma^{D, m, l}\right]-\widetilde{G}\left(\tilde{x}, D \psi(t, \tilde{x}), D^{2} \psi(t, \tilde{x})\right)\left((l+1) \delta_{m} \wedge \delta-l \delta_{m}\right)\right|\right] \\
& \leq \hat{\mathbb{E}}\left[\left|\hat{\mathbb{E}}_{l \delta_{m}}\left[\left\langle D \psi(t, \tilde{x}), \xi^{D, 1, m, l}\right\rangle+\gamma^{D, m, l}\right]-\widetilde{G}\left(\widetilde{X}_{l \delta_{m}}^{\varepsilon_{m}, x}, D \psi(t, \tilde{x}), D^{2} \psi(t, \tilde{x})\right)\left((l+1) \delta_{m} \wedge \delta-l \delta_{m}\right)\right|\right] \\
& \quad+\hat{\mathbb{E}}\left[\left|\widetilde{G}\left(\widetilde{X}_{l \delta_{m}}^{\varepsilon_{m}, x}, D \psi(t, \tilde{x}), D^{2} \psi(t, \tilde{x})\right)-\widetilde{G}\left(\tilde{x}, D \psi(t, \tilde{x}), D^{2} \psi(t, \tilde{x})\right)\right|\right]\left((l+1) \delta_{m} \wedge \delta-l \delta_{m}\right) \\
& \quad \leq C\left(L_{1}, L_{2}, \eta, \psi\right)\left(1+|x|^{3}\right)\left(\varepsilon_{m}+\sqrt{\delta}\left((l+1) \delta_{m} \wedge \delta-l \delta_{m}\right)\right) .
\end{aligned}
$$

It follows that

$$
\begin{aligned}
& \left|\hat{\mathbb{E}}\left[\sum_{l=0}^{N^{m}} J^{D, m, l}\right]+H(t, \tilde{x}, \psi) \delta\right|=\left|\hat{\mathbb{E}}\left[\sum_{l=0}^{N^{m}-1} J^{D, m, l}+\hat{\mathbb{E}}_{N^{m} \delta}\left[J^{D, m, N^{m}}\right]\right]+H(t, \tilde{x}, \psi) \delta\right| \\
& \leq\left|\hat{\mathbb{E}}\left[\sum_{l=0}^{N^{m}-1} J^{D, m, l}\right]+H(t, \tilde{x}, \psi) N^{m} \delta_{m}\right|+\hat{\mathbb{E}}\left[\left|\hat{\mathbb{E}}_{N^{m} \delta}\left[J^{D, m, N^{m}}\right]+H(t, \tilde{x}, \psi)\left(\delta-N^{m} \delta_{m}\right)\right|\right] \\
& \leq \cdots \ldots \ldots \\
& \leq \sum_{l=0}^{N^{m}} \hat{\mathbb{E}}\left[\left|\hat{\mathbb{E}}_{l \delta_{m}}\left[J^{D, m, l}\right]+H(t, \tilde{x}, \psi)\left((l+1) \delta_{m} \wedge \delta-l \delta_{m}\right)\right|\right] \leq C\left(L_{1}, L_{2}, \eta, \psi\right)\left(1+|x|^{3}\right)\left(\frac{\varepsilon_{m}}{\delta_{m}}+\sqrt{\delta}\right) \delta .
\end{aligned}
$$


Consequently, in view of the inequality (23), we deduce that

$$
\left|\hat{\mathbb{E}}\left[J^{m}\right]+H(t, \tilde{x}, \psi) \delta\right| \leq C\left(L_{1}, L_{2}, \eta, \psi\right)\left(1+|x|^{3}\right)\left(\frac{\varepsilon_{m}}{\delta_{m}}+\sqrt{\delta_{m}}+\sqrt{\delta}+\sqrt{\rho_{m}}\right) \delta .
$$

The proof is complete.

Remark 4.6 The assumption (H3) is used to establish a uniform $L_{G}^{3}$-estimate of the slow diffusion process, which is crucial for inequality (20) in our setting. Indeed, one could strengthen the assumption (H2) to remove (H3); see Lemma 3.2 in [23].

Lemma 4.7 Suppose that $(p, A)$ is in $\mathbb{R}^{n} \times \mathbb{S}(n)$. Then, for each $s \in\left[l \delta_{m},(l+1) \delta_{m} \wedge \delta\right], l=0, \ldots, N^{m}$, it holds that

$$
\begin{aligned}
& \left|\hat{\mathbb{E}}_{l \delta_{m}}\left[\int_{l \delta_{m}}^{s}\left\langle p, \widetilde{b}\left(\widetilde{X}_{l \delta_{m}}^{\varepsilon_{m}, x}, \bar{X}_{r}^{D, \varepsilon_{m}, x}\right)\right\rangle d r+\frac{1}{2} \int_{l \delta_{m}}^{s} \widetilde{\sigma}^{A}\left(\widetilde{X}_{l \delta_{m}}^{\varepsilon_{m}, x}, \bar{X}_{r}^{D, \varepsilon_{m}, x}\right) d\langle B\rangle_{r}\right]-\widetilde{G}\left(\widetilde{X}_{l \delta_{m}}^{\varepsilon, x}, p, A\right)\left(s-l \delta_{m}\right)\right| \\
& \leq C\left(L_{1}, L_{2}, \eta\right)(|p|+|A|)\left(1+\left|\widetilde{X}_{l \delta_{m}}^{\varepsilon_{m}, x}\right|^{2}+\left|\bar{X}_{l \delta_{m}}^{\varepsilon_{m}, x}\right|^{2}\right) \varepsilon_{m} .
\end{aligned}
$$

Proof. Consider the following $G$-SDE: for each $x=(\tilde{x}, \bar{x}) \in \mathbb{R}^{2 n}$,

$$
\bar{X}_{t}^{\prime \prime \varepsilon,(\tilde{x}, \bar{x})}=\bar{x}+\int_{0}^{t} \frac{\bar{b}\left(\tilde{x}, \bar{X}_{r}^{\prime, \varepsilon,(\tilde{x}, \bar{x})}\right)}{\varepsilon} d r+\int_{0}^{t} \frac{\bar{\sigma}\left(\tilde{x}, \bar{X}_{r}^{\prime, \varepsilon,(\tilde{x}, \bar{x})}\right)}{\sqrt{\varepsilon}} d B_{r}
$$

Recalling equation (5), we get that

$$
\bar{X}_{\frac{t}{\varepsilon_{m}}}^{(\tilde{x}, \bar{x})}=\bar{x}+\frac{1}{\varepsilon_{m}} \int_{0}^{t} \bar{b}\left(\tilde{x}, \bar{X}_{\frac{r}{\varepsilon_{m}}}^{(\tilde{x}, \bar{x})}\right) d r+\frac{1}{\sqrt{\varepsilon_{m}}} \int_{0}^{t} \bar{\sigma}\left(\tilde{x}, \bar{X}_{\frac{r}{\varepsilon_{m}}}^{(\tilde{x}, \bar{x})}\right) d B_{r}^{\varepsilon_{m}},
$$

where $\left(B_{r}^{\varepsilon_{m}}=\sqrt{\varepsilon_{m}} B_{\frac{r}{\varepsilon_{m}}}\right)_{r \geq 0}$ also is a $G$-Brownian motion. Then, by a standard approximation method, we derive that $\left(\bar{X}_{t}^{\prime, \varepsilon_{m},(\tilde{x}, \bar{x})}, B_{t}\right)$ has the same distribution as $\left(\bar{X}_{\frac{t}{\varepsilon_{m}}}^{(\tilde{x}, \bar{x})}, B_{t}^{\varepsilon_{m}}\right)$. It follows that

$$
\begin{aligned}
& \hat{\mathbb{E}}\left[\int_{0}^{t}\left\langle p, \widetilde{b}\left(\tilde{x}, \bar{X}_{r}^{\prime, \varepsilon_{m},(\tilde{x}, \bar{x})}\right)\right\rangle d r+\frac{1}{2} \sum_{i, j=1}^{d} \int_{0}^{t} \widetilde{\sigma}_{i j}^{A}\left(\tilde{x}, \bar{X}_{r}^{\prime, \varepsilon_{m},(\tilde{x}, \bar{x})}\right) d\left\langle B^{i}, B^{j}\right\rangle_{r}\right] \\
& =\hat{\mathbb{E}}\left[\int_{0}^{t}\left\langle p, \widetilde{b}\left(\tilde{x}, \bar{X}_{\frac{r}{\varepsilon_{m}}}^{(\tilde{x}, \bar{x})}\right)\right\rangle d r+\frac{1}{2} \sum_{i, j=1}^{d} \int_{0}^{t} \widetilde{\sigma}_{i j}^{A}\left(\bar{X}_{\frac{r}{\varepsilon_{m}}}^{(\tilde{x}, \bar{x})}\right) d\left\langle B^{\varepsilon_{m}, i}, B^{\varepsilon_{m}, j}\right\rangle_{r}\right] \\
& =\varepsilon_{m} \hat{\mathbb{E}}\left[\int_{0}^{\frac{t}{\varepsilon_{m}}}\left\langle p, \widetilde{b}\left(\tilde{x}, \bar{X}_{r}^{x}\right)\right\rangle d r+\frac{1}{2} \sum_{i, j=1}^{d} \int_{0}^{\frac{t}{\varepsilon_{m}}} \widetilde{\sigma}_{i j}^{A}\left(\tilde{x}, \bar{X}_{r}^{x}\right) d\left\langle B^{i}, B^{j}\right\rangle_{r}\right],
\end{aligned}
$$

which together with inequality (7) yields that, for each $t>0$,

$$
\begin{aligned}
& \left|\hat{\mathbb{E}}\left[\int_{0}^{t}\left\langle p, \widetilde{b}\left(\tilde{x}, \bar{X}_{r}^{\prime, \varepsilon_{m},(\tilde{x}, \bar{x})}\right)\right\rangle d r+\frac{1}{2} \sum_{i, j=1}^{d} \int_{0}^{t} \tilde{\sigma}_{i j}^{A}\left(\tilde{x}, \bar{X}_{r}^{\prime, \varepsilon_{m},(\tilde{x}, \bar{x})}\right) d\left\langle B^{i}, B^{j}\right\rangle_{r}\right]-\widetilde{G}(\tilde{x}, p, A) t\right| \\
& \leq\left|\hat{\mathbb{E}}\left[\int_{0}^{\frac{t}{\varepsilon_{m}}}\left\langle p, \widetilde{b}\left(\tilde{x}, \bar{X}_{r}^{x}\right)\right\rangle d r+\frac{1}{2} \sum_{i, j=1}^{d} \int_{0}^{\frac{t}{\varepsilon_{m}}} \widetilde{\sigma}_{i j}^{A}\left(\tilde{x}, \bar{X}_{r}^{x}\right) d\left\langle B^{i}, B^{j}\right\rangle_{r}\right]-\widetilde{G}(\tilde{x}, p, A) \frac{t}{\varepsilon_{m}}\right| \varepsilon_{m} \\
& \leq C\left(L_{1}, L_{2}, \eta\right)(|p|+|A|)\left(1+|\tilde{x}|^{2}+|\bar{x}|^{2}\right) \varepsilon_{m} .
\end{aligned}
$$


On the other hand, recalling the definition $\bar{X}^{D, \varepsilon_{m}, x}$ and using the Markov property (see Assertion (7) of Theorem 5.1 in [22]), we conclude that

$$
\begin{aligned}
& \hat{\mathbb{E}}_{l \delta_{m}}\left[\int_{l \delta_{m}}^{s}\left\langle p, \widetilde{b}\left(\widetilde{X}_{l \delta_{m}}^{\varepsilon_{m}, x}, \bar{X}_{r}^{D, \varepsilon_{m}, x}\right)\right\rangle d r+\frac{1}{2} \sum_{i, j=1}^{d} \int_{l \delta_{m}}^{s} \widetilde{\sigma}_{i j}^{A}\left(\widetilde{X}_{l \delta_{m}}^{\varepsilon_{m}, x}, \bar{X}_{r}^{D, \varepsilon_{m}, x}\right) d\left\langle B^{i}, B^{j}\right\rangle_{r}\right] \\
& =\hat{\mathbb{E}}\left[\int_{0}^{s-l \delta_{m}}\left\langle p, \widetilde{b}\left(\tilde{x}^{\prime}, \bar{X}_{r}^{\prime, \varepsilon_{m},\left(\tilde{x}^{\prime}, \bar{x}^{\prime}\right)}\right)\right\rangle d r+\frac{1}{2} \int_{0}^{s-l \delta_{m}} \widetilde{\sigma}^{A}\left(\tilde{x}^{\prime}, \bar{X}_{r}^{\prime, \varepsilon_{m},\left(\tilde{x}^{\prime}, \bar{x}^{\prime}\right)}\right) d\langle B\rangle_{r}\right]_{\left(\tilde{x}^{\prime}, \bar{x}^{\prime}\right)=\left(\tilde{X}_{l \delta_{m}, x}^{\varepsilon_{m}, X_{X}}, \bar{X}_{l \delta_{m}}^{\varepsilon_{m}, x}\right)},
\end{aligned}
$$

which together with the inequality (25) indicates the desired result. This ends the proof.

Finally, we are ready to state the proofs of Theorem 3.6 and Theorem 3.9 .

The proof of Theorem 3.6. Without loss of generality, assume that $t \in[0, T]$. Let $(t, \tilde{x}, \bar{x}) \in$ $[0, T] \times \mathbb{R}^{2 n}$ be fixed. Denote by $\widetilde{u}^{\varphi}$ the solution to PDE (9) with the initial condition $\varphi$. Similarly, we can define $u^{\varphi, \varepsilon}$. The proof is divided into the following two steps.

Step $1\left(\varphi \in C_{b . l i p}\left(\mathbb{R}^{n}\right)\right)$. Suppose that the sequence $\left(\varepsilon_{l}\right)_{l \geq 1}$ converges to 0 . Then, from Lemma4.2, we can find a subsequence $\left(\varepsilon_{l_{m}}\right)_{m>1}$ such that $u^{\varphi, \varepsilon_{l_{m}}}$ converges to some function $\widetilde{u}^{\varphi, *} \in C_{b}\left([0, T] \times \mathbb{R}^{n}\right)$ on $[0, T] \times \mathbb{R}^{2 n}$. Applying Lemma 4.5 yields that $\widetilde{u}^{\varphi, *}$ is a viscosity solution to the averaged PDE (9). It follows from Lemma B.1 in appendix B that $\widetilde{u}^{\varphi, *} \equiv \widetilde{u}^{\varphi}$. Thus, we derive that

$$
\lim _{m \rightarrow \infty} u^{\varphi, \varepsilon_{l_{m}}}(t, \tilde{x}, \bar{x})=\widetilde{u}^{\varphi}(t, \tilde{x})
$$

which implies the desired result.

Step $2\left(\varphi \in C\left(\mathbb{R}^{n}\right)\right.$ of polynomial growth). For each positive integer $N$, we can find a function $\varphi_{N} \in C_{b . l i p}\left(\mathbb{R}^{n}\right)$ so that

$$
\left|\varphi_{N}\left(\tilde{x}^{\prime}\right)-\varphi\left(\tilde{x}^{\prime}\right)\right| \leq C(\varphi) \frac{1+\left|\tilde{x}^{\prime}\right| C(\varphi)}{N}, \forall \tilde{x}^{\prime} \in \mathbb{R}^{n}
$$

With the help of Lemma 4.3, we have that, for any $\left(s, x^{\prime}\right) \in[0, T] \times \mathbb{R}^{2 n}$,

$$
\hat{\mathbb{E}}\left[\left|\varphi_{N}\left(\widetilde{X}_{s}^{\varepsilon, x^{\prime}}\right)-\varphi\left(\widetilde{X}_{s}^{\varepsilon, x^{\prime}}\right)\right|\right] \leq C(\varphi) \frac{1+\hat{\mathbb{E}}\left[\left|\widetilde{X}_{s}^{\varepsilon, x^{\prime}}\right|^{C(\varphi)}\right]}{N} \leq C\left(L_{1}, L_{2}, T, \varphi\right) \frac{1+\left|\tilde{x}^{\prime}\right|^{C(\varphi)}}{N} .
$$

Then, from inequality (26), we get that

$$
\begin{aligned}
& \liminf _{\varepsilon \rightarrow 0} \hat{\mathbb{E}}\left[\varphi\left(\widetilde{X}_{s}^{\varepsilon, x^{\prime}}\right)\right] \geq-C\left(L_{1}, L_{2}, T, \varphi\right) \frac{1+\left|\tilde{x}^{\prime}\right|^{C(\varphi)}}{N}+\lim _{\varepsilon \rightarrow 0} \hat{\mathbb{E}}\left[\varphi_{N}\left(\widetilde{X}_{s}^{\varepsilon, x^{\prime}}\right)\right], \\
& \limsup _{\varepsilon \rightarrow 0} \hat{\mathbb{E}}\left[\varphi\left(\widetilde{X}_{s}^{\varepsilon, x^{\prime}}\right)\right] \leq C\left(L_{1}, L_{2}, T, \varphi\right) \frac{1+\left|\tilde{x}^{\prime}\right|^{C(\varphi)}}{N}+\lim _{\varepsilon \rightarrow 0} \hat{\mathbb{E}}\left[\varphi_{N}\left(\widetilde{X}_{s}^{\varepsilon, x^{\prime}}\right)\right],
\end{aligned}
$$

which implies that

$$
\limsup _{\varepsilon \rightarrow 0} \hat{\mathbb{E}}\left[\varphi\left(\widetilde{X}_{s}^{\varepsilon, x^{\prime}}\right)\right] \leq \liminf _{\varepsilon \rightarrow 0} \hat{\mathbb{E}}\left[\varphi\left(\widetilde{X}_{s}^{\varepsilon, x^{\prime}}\right)\right]+C\left(L_{1}, L_{2}, T, \varphi\right) \frac{1+\left|\tilde{x}^{\prime}\right|^{C(\varphi)}}{N} .
$$

Sending $N \rightarrow \infty$, we deduce that $\lim _{\varepsilon \rightarrow 0} \hat{\mathbb{E}}\left[\varphi\left(\widetilde{X}_{s}^{\varepsilon, x^{\prime}}\right)\right]$ exists. Moreover, recalling equation (26), we obtain that

$$
\lim _{\varepsilon \rightarrow 0} \hat{\mathbb{E}}\left[\varphi\left(\widetilde{X}_{s}^{\varepsilon, x^{\prime}}\right)\right]=\lim _{N \rightarrow \infty} \lim _{\varepsilon \rightarrow 0} \hat{\mathbb{E}}\left[\varphi_{N}\left(\widetilde{X}_{s}^{\varepsilon, x^{\prime}}\right)\right]=\lim _{N \rightarrow \infty} \widetilde{u}^{\varphi_{N}}\left(s, \tilde{x}^{\prime}\right)=: \widetilde{u}^{\varphi, *}\left(s, \tilde{x}^{\prime}\right) .
$$


On the other hand, applying equation (26) again, we get that

$$
\left|\widetilde{u}^{\varphi_{N}}\left(s, \tilde{x}^{\prime}\right)-\widetilde{u}^{\varphi, *}\left(s, \tilde{x}^{\prime}\right)\right|=\lim _{\varepsilon \rightarrow 0}\left|\hat{\mathbb{E}}\left[\varphi_{N}\left(\widetilde{X}_{s}^{\varepsilon, x^{\prime}}\right)\right]-\hat{\mathbb{E}}\left[\varphi\left(\widetilde{X}_{s}^{\varepsilon, x^{\prime}}\right)\right]\right| \leq C(\varphi) \frac{1+\left|\tilde{x}^{\prime}\right|^{C(\varphi)}}{N},
$$

which indicates that $\widetilde{u}^{\varphi_{N}}$ converges uniformly to $\widetilde{u}^{\varphi, *}$ on each compact subset of $[0, T] \times \mathbb{R}^{n}$. In the spirit of Proposition 4.3 in [9] and Lemma B.1 in appendix B, we conclude that $\widetilde{u}^{\varphi, *}$ is the unique viscosity solution to the averaged PDE (9). The proof is complete.

The proof of Theorem 3.9. It suffices to prove the case that $k=2$, since other cases can be proved by iterative method. Without loss of generality, assume that $t_{1}, t_{2} \in[0, T]$.

From Theorem 3.6 and Lemma 4.3, it is easy to check that $\varphi^{1}\left(\tilde{x}^{1}\right)=\lim _{\varepsilon \rightarrow 0} \hat{\mathbb{E}}\left[\varphi\left(\tilde{x}^{1}, \widetilde{X}_{t_{2}-t_{1}}^{\varepsilon,\left(\tilde{x}^{1}, 0\right)}\right)\right]$ is well-defined and of polynomial growth. We claim that

$$
\left(\tilde{x}^{1}, \bar{x}^{1}\right) \rightarrow \hat{\mathbb{E}}\left[\varphi\left(\tilde{x}^{1}, \widetilde{X}_{t_{2}-t_{1}}^{\varepsilon,\left(\tilde{x}^{1}, \bar{x}^{1}\right)}\right)\right] \text { uniformly converges to } \varphi^{1}\left(\tilde{x}^{1}\right) \text { on each compact subset of } \mathbb{R}^{2 n},
$$

whose proof will be given later.

According to the Markov property (see 22]) and Lemma 4.3 we conclude that,

$$
\begin{aligned}
& \left|\hat{\mathbb{E}}\left[\varphi\left(\widetilde{X}_{t_{1}}^{\varepsilon, x}, \widetilde{X}_{t_{2}}^{\varepsilon, x}\right)\right]-\hat{\mathbb{E}}\left[\varphi^{1}\left(\widetilde{X}_{t_{1}}^{\varepsilon, x}\right)\right]\right| \leq \hat{\mathbb{E}}\left[\left|\hat{\mathbb{E}}\left[\varphi\left(\tilde{x}^{1}, \widetilde{X}_{t_{2}-t_{1}}^{\left.\varepsilon, \tilde{x}^{1}, \bar{x}^{1}\right)}\right)\right]_{\left(\tilde{x}^{1}, \bar{x}^{1}\right)=\left(\tilde{X}_{t_{1}}^{\varepsilon, x}, \bar{X}_{t_{1}}^{\varepsilon, x}\right)}-\varphi^{1}\left(\widetilde{X}_{t_{1}}^{\varepsilon, x}\right)\right|\right] \\
& \leq \sup _{\left|\tilde{x}^{1}\right|,\left|\tilde{x}^{1}\right| \leq N}\left|\hat{\mathbb{E}}\left[\varphi\left(\tilde{x}^{1}, \tilde{X}_{t_{2}-t_{1}}^{\varepsilon,\left(\tilde{x}^{1}, \bar{x}^{1}\right)}\right)\right]-\varphi^{1}\left(\tilde{x}^{1}\right)\right|+\frac{C\left(L_{1}, L_{2}, T, \varphi\right)}{N} \hat{\mathbb{E}}\left[\left(1+\left|\widetilde{X}_{t_{1}}^{\varepsilon, x}\right|^{C(\varphi)}+\left|\bar{X}_{t_{1}}^{\varepsilon, x}\right|\right)\right] \\
& \leq \sup _{\left|\tilde{x}^{1}\right|,\left|\bar{x}^{1}\right| \leq N}\left|\hat{\mathbb{E}}\left[\varphi\left(\tilde{x}^{1}, \widetilde{X}_{t_{2}-t_{1}}^{\varepsilon,\left(\tilde{x}^{1}, \bar{x}^{1}\right)}\right)\right]-\varphi^{1}\left(\tilde{x}^{1}\right)\right|+\frac{C\left(L_{1}, L_{2}, T, \varphi\right)}{N}\left(1+|x|^{C(\varphi)}\right), \quad \forall N \geq 1,
\end{aligned}
$$

where we have used Lemma 4.1 in the last inequality. It follows that

$$
\limsup _{\varepsilon \rightarrow 0}\left|\hat{\mathbb{E}}\left[\varphi\left(\widetilde{X}_{t_{1}}^{\varepsilon, x}, \widetilde{X}_{t_{2}}^{\varepsilon, x}\right)\right]-\hat{\mathbb{E}}\left[\varphi^{1}\left(\widetilde{X}_{t_{1}}^{\varepsilon, x}\right)\right]\right| \leq \frac{C\left(L_{1}, L_{2}, T, \varphi\right)}{N}\left(1+|x|^{C(\varphi)}\right) .
$$

Sending $N \rightarrow 0$, we obtain that

$$
\lim _{\varepsilon \rightarrow 0} \hat{\mathbb{E}}\left[\varphi\left(\widetilde{X}_{t_{1}}^{\varepsilon, x}, \widetilde{X}_{t_{2}}^{\varepsilon, x}\right)\right]=\lim _{\varepsilon \rightarrow 0} \hat{\mathbb{E}}\left[\varphi^{1}\left(\widetilde{X}_{t_{1}}^{\varepsilon, x}\right)\right],
$$

which is the desired result.

Now, we shall prove the above claim. For each positive integer $N$, we can find a function $\varphi_{N} \in$ $C_{b . l i p}\left(\mathbb{R}^{2 n}\right)$ so that

$$
\left|\varphi_{N}\left(\tilde{x}^{1}, \tilde{x}^{2}\right)-\varphi\left(\tilde{x}^{1}, \tilde{x}^{2}\right)\right| \leq C(\varphi) \frac{1+\left|\tilde{x}^{1}\right|^{C(\varphi)}+\left|\tilde{x}^{2}\right|^{C(\varphi)}}{N}, \forall \tilde{x}^{1}, \tilde{x}^{2} \in \mathbb{R}^{n} .
$$

Thus, according to Lemma 4.3 we have that

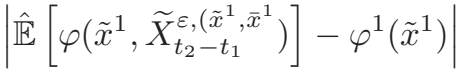

$$
\begin{aligned}
& \leq\left|\hat{\mathbb{E}}\left[\varphi\left(\tilde{x}^{1}, \widetilde{X}_{t_{2}-t_{1}}^{\varepsilon,\left(\tilde{x}^{1}, \bar{x}^{1}\right)}\right)\right]-\hat{\mathbb{E}}\left[\varphi_{N}\left(\tilde{x}^{1}, \widetilde{X}_{t_{2}-t_{1}}^{\varepsilon,\left(\tilde{x}^{1}, \bar{x}^{1}\right)}\right)\right]\right|+\left|\hat{\mathbb{E}}\left[\varphi_{N}\left(\tilde{x}^{1}, \widetilde{X}_{t_{2}-t_{1}}^{\varepsilon,\left(\tilde{x}^{1}, \bar{x}^{1}\right)}\right)\right]-\varphi_{N}^{1}\left(\tilde{x}^{1}\right)\right|+\left|\varphi_{N}^{1}\left(\tilde{x}^{1}\right)-\varphi^{1}\left(\tilde{x}^{1}\right)\right| \\
& \leq\left|\hat{\mathbb{E}}\left[\varphi_{N}\left(\tilde{x}^{1}, \widetilde{X}_{t_{2}-t_{1}}^{\left.\varepsilon, \tilde{x}^{1}, \bar{x}^{1}\right)}\right)\right]-\varphi_{N}^{1}\left(\tilde{x}^{1}\right)\right|+C\left(L_{1}, L_{2}, T, \varphi\right) \frac{1+\left|\tilde{x}^{1}\right|^{C(\varphi)}}{N} .
\end{aligned}
$$

On the other hand, by a similar analysis as in Lemma 4.2. it is easy to check that $\hat{\mathbb{E}}\left[\varphi_{N}\left(\tilde{x}^{1}, \widetilde{X}_{t_{2}, t_{1}}^{\left.\varepsilon, \tilde{x}^{1}, \bar{x}^{1}\right)}\right)\right]$ uniformly converges to $\varphi_{N}^{1}\left(\tilde{x}^{1}\right)$ on each compact subset of $\mathbb{R}^{2 n}$. Consequently, from the above inequality, we get that the desired claim holds. The proof is complete. 


\section{Extension}

In the previous sections, we develop a useful approach to the study of averaging of $G$-SDEs with two time scales by using nonlinear stochastic analysis and viscosity solution theory. We would like to mention that our main ideas carry over to much more general frameworks. In this section, we will extend the previous results to a more general case.

Consider the following fast-slow scale diffusion process (see [33]):

$$
\left\{\begin{aligned}
\widetilde{S}_{t}^{\varepsilon, x}=\tilde{x} & +\int_{0}^{t} \widetilde{b}\left(\widetilde{S}_{s}^{\varepsilon, x}, \bar{S}_{s}^{\varepsilon, x}\right) d s+\sum_{i, j=1}^{d} \int_{0}^{t} \widetilde{h}_{i j}\left(\widetilde{S}_{s}^{\varepsilon, x}, \bar{S}_{s}^{\varepsilon, x}\right) d\left\langle B^{i}, B^{j}\right\rangle_{s}+\int_{0}^{t} \widetilde{\sigma}\left(\widetilde{S}_{s}^{\varepsilon, x}, \bar{S}_{s}^{\varepsilon, x}\right) d B_{s}, \\
\bar{S}_{t}^{\varepsilon, x}= & +\int_{0}^{t}\left(\frac{\bar{b}\left(\widetilde{S}_{s}^{\varepsilon, x}, \bar{S}_{s}^{\varepsilon, x}\right)}{\varepsilon}+\frac{\bar{b}^{1}\left(\widetilde{S}_{s}^{\varepsilon, x}, \bar{S}_{s}^{\varepsilon, x}\right)}{\sqrt{\varepsilon}}+\bar{b}^{2}\left(\widetilde{S}_{s}^{\varepsilon, x}, \bar{S}_{s}^{\varepsilon, x}\right)\right) d s \\
& +\sum_{i, j=1}^{d} \int_{0}^{t}\left(\frac{\bar{h}_{i j}\left(\widetilde{S}_{s}^{\varepsilon, x}, \bar{S}_{s}^{\varepsilon, x}\right)}{\varepsilon}+\frac{\bar{h}_{i j}^{1}\left(\widetilde{S}_{s}^{\varepsilon, x}, \bar{S}_{s}^{\varepsilon, x}\right)}{\varepsilon}+\bar{h}_{i j}^{2}\left(\widetilde{S}_{s}^{\varepsilon, x}, \bar{S}_{s}^{\varepsilon, x}\right)\right) d\left\langle B^{i}, B^{j}\right\rangle_{s} \\
& +\int_{0}^{t}\left(\frac{\bar{\sigma}\left(\widetilde{S}_{s}^{\varepsilon, x}, \bar{S}_{s}^{\varepsilon, x}\right)}{\sqrt{\varepsilon}}+\bar{\sigma}^{1}\left(\widetilde{S}_{s}^{\varepsilon, x}, \bar{S}_{s}^{\varepsilon, x}\right)\right) d B_{s},
\end{aligned}\right.
$$

for each $x=(\tilde{x}, \bar{x}) \in \mathbb{R}^{2 n}$, where $\bar{b}^{1}, \bar{b}^{2}, \bar{h}_{i j}^{1}=\bar{h}_{j i}^{1}, \bar{h}_{i j}^{2}=\bar{h}_{j i}^{2}: \mathbb{R}^{2 n} \rightarrow \mathbb{R}^{n}, \bar{\sigma}^{1}: \mathbb{R}^{2 n} \rightarrow \mathbb{R}^{n \times d}$ are deterministic non-periodic functions satisfying the following.

(H4) There exists a constant $L_{1}>0$ such that, for any $x, x^{\prime} \in \mathbb{R}^{2 n}$,

$$
\left|\ell(x)-\ell\left(x^{\prime}\right)\right| \leq L_{1}\left|x-x^{\prime}\right| \text { and }|\ell(0)| \leq L_{1} \text {, for } \ell=\bar{b}^{1}, \bar{b}^{2}, \bar{h}_{i j}^{1}, \bar{h}_{i j}^{2} \text {, and } \bar{\sigma}^{1} .
$$

Note that the new coefficients added vary slowly compared with the original ones, and therefore they do not affect the structure of the averaged PDEs as in [33]; see Theorem [5.2

Lemma 5.1 Assume (H1), (H2) and (H4) hold. Then, there exists a constant $C\left(L_{1}, \eta, T\right)$, such that for each $x \in \mathbb{R}^{2 n}$,

(i) $\hat{\mathbb{E}}\left[\sup _{0 \leq t \leq T}\left|\widetilde{S}_{t}^{\varepsilon, x}\right|^{2}\right]+\sup _{0 \leq t \leq T} \hat{\mathbb{E}}\left[\left|\bar{S}_{t}^{\varepsilon, x}\right|^{2}\right] \leq C\left(L_{1}, \eta, T\right)\left(1+|x|^{2}\right)$,

(ii) $\hat{\mathbb{E}}\left[\sup _{0 \leq t \leq T}\left|\widetilde{S}_{t}^{\varepsilon, x}-\widetilde{X}_{t}^{\varepsilon, x}\right|^{2}\right] \leq C\left(L_{1}, \eta, T\right)\left(1+|x|^{2}\right) \varepsilon$.

Proof. We shall only give the sketch of the proof for readers' convenience. Without loss of generality, assume that $\widetilde{h}_{i j}=\bar{h}_{i j}=\bar{h}_{i j}^{1}=\bar{h}_{i j}^{2}=0$, for $i, j=1, \ldots, d$. The proof is divided into the following two steps.

Step 1 (Assertion (i)). Applying $G$-Itô's formula, we deduce that

$$
\begin{aligned}
e^{\frac{\eta}{\varepsilon}} t & \left|\bar{S}_{t}^{\varepsilon, x}\right|^{2}-|\bar{x}|^{2}-M_{t}^{1}-\frac{\eta}{\varepsilon} \int_{0}^{t} e^{\frac{\eta}{\varepsilon} s}\left|\bar{S}_{s}^{\varepsilon, x}\right|^{2} d s \\
\leq & \frac{2}{\varepsilon} \int_{0}^{t} e^{\frac{\eta}{\varepsilon} s}\left[\left\langle\bar{S}_{s}^{\varepsilon, x}, \bar{b}\left(\widetilde{S}_{s}^{\varepsilon, x}, \bar{S}_{s}^{\varepsilon, x}\right)\right\rangle+G\left(\left(\bar{\sigma}\left(\widetilde{S}_{s}^{\varepsilon, x}, \bar{S}_{s}^{\varepsilon, x}\right)\right)^{\top} \bar{\sigma}\left(\widetilde{S}_{s}^{\varepsilon, x}, \bar{S}_{s}^{\varepsilon, x}\right)\right)\right] d s \\
& +\frac{2}{\sqrt{\varepsilon}} \int_{0}^{t} e^{\frac{\eta}{\varepsilon} s}\left[\left\langle\bar{S}_{s}^{\varepsilon, x}, \bar{b}^{1}\left(\widetilde{S}_{s}^{\varepsilon, x}, \bar{S}_{s}^{\varepsilon, x}\right)\right\rangle+G\left(\left(\left(\bar{\sigma}^{1}\right)^{\top} \bar{\sigma}+(\bar{\sigma})^{\top} \bar{\sigma}^{1}\right)\left(\widetilde{S}_{s}^{\varepsilon, x}, \bar{S}_{s}^{\varepsilon, x}\right)\right)\right] d s \\
& +2 \int_{0}^{t} e^{\frac{\eta}{\varepsilon} s}\left[\left\langle\bar{S}_{s}^{\varepsilon, x}, \bar{b}^{2}\left(\widetilde{S}_{s}^{\varepsilon, x}, \bar{S}_{s}^{\varepsilon, x}\right)\right\rangle+G\left(\left(\bar{\sigma}^{1}\left(\widetilde{S}_{s}^{\varepsilon, x}, \bar{S}_{s}^{\varepsilon, x}\right)\right)^{\top} \bar{\sigma}^{1}\left(\widetilde{S}_{s}^{\varepsilon, x}, \bar{S}_{s}^{\varepsilon, x}\right)\right)\right] d s,
\end{aligned}
$$


where $M_{t}^{1}$ is a symmetric $G$-martingale with $M_{0}^{1}=0$.

Recalling assumptions (H1) and (H2), we could get that

$$
\begin{aligned}
& 2\left\langle\bar{S}_{s}^{\varepsilon, x}, \bar{b}\left(\widetilde{S}_{s}^{\varepsilon, x}, \bar{S}_{s}^{\varepsilon, x}\right)\right\rangle+2 G\left(\left(\bar{\sigma}\left(\widetilde{S}_{s}^{\varepsilon, x}, \bar{S}_{s}^{\varepsilon, x}\right)\right)^{\top}\left(\bar{\sigma}\left(\widetilde{S}_{s}^{\varepsilon, x}, \bar{S}_{s}^{\varepsilon, x}\right)\right)\right) \\
& \leq-\frac{3}{2} \eta\left|\bar{S}_{s}^{\varepsilon, x}\right|^{2}+C\left(L_{1}, \eta\right)\left(1+\left|\widetilde{S}_{s}^{\varepsilon, x}\right|^{2}\right),
\end{aligned}
$$

and

$$
\begin{aligned}
& 2\left\langle\bar{S}_{s}^{\varepsilon, x}, \bar{b}^{1}\left(\widetilde{S}_{s}^{\varepsilon, x}, \bar{S}_{s}^{\varepsilon, x}\right)\right\rangle+2 G\left(\left(\left(\bar{\sigma}^{1}\right)^{\top} \bar{\sigma}+(\bar{\sigma})^{\top} \bar{\sigma}^{1}\right)\left(\widetilde{S}_{s}^{\varepsilon, x}, \bar{S}_{s}^{\varepsilon, x}\right)\right) \leq C\left(L_{1}\right)\left(1+\left|\bar{S}_{s}^{\varepsilon, x}\right|^{2}+\left|\widetilde{S}_{s}^{\varepsilon, x}\right|^{2}\right), \\
& 2\left\langle\bar{S}_{s}^{\varepsilon, x}, \bar{b}^{2}\left(\widetilde{S}_{s}^{\varepsilon, x}, \bar{S}_{s}^{\varepsilon, x}\right)\right\rangle+2 G\left(\left(\bar{\sigma}^{1}\left(\widetilde{S}_{s}^{\varepsilon, x}, \bar{S}_{s}^{\varepsilon, x}\right)\right)^{\top} \bar{\sigma}^{1}\left(\widetilde{S}_{s}^{\varepsilon, x}, \bar{S}_{s}^{\varepsilon, x}\right)\right) \leq C\left(L_{1}\right)\left(1+\left|\bar{S}_{s}^{\varepsilon, x}\right|^{2}+\left|\widetilde{S}_{s}^{\varepsilon, x}\right|^{2}\right) .
\end{aligned}
$$

It follows from inequality (27) that

$e^{\frac{\eta}{\varepsilon} t}\left|\bar{S}_{t}^{\varepsilon, x}\right|^{2} \leq|\bar{x}|^{2}+M_{t}^{1}+\frac{1}{2 \varepsilon}\left(C\left(L_{1}\right)(\sqrt{\varepsilon}+\varepsilon)-\eta\right) \int_{0}^{t} e^{\frac{\eta}{\varepsilon} s}\left|\bar{S}_{s}^{\varepsilon, x}\right|^{2} d s+\frac{C\left(L_{1}, \eta\right)}{\varepsilon} \int_{0}^{t} e^{\frac{\eta}{\varepsilon} s}\left(1+\left|\widetilde{S}_{s}^{\varepsilon, x}\right|^{2}\right) d s$

Thus, if $\varepsilon \leq \frac{\eta^{2}}{C\left(L_{1}\right)}$, we could obtain that

$$
\hat{\mathbb{E}}\left[\left|\bar{S}_{t}^{\varepsilon, x}\right|^{2}\right] \leq e^{-\frac{\eta}{\varepsilon} t}|\bar{x}|^{2}+\frac{C\left(L_{1}, \eta\right)}{\varepsilon} \int_{0}^{t} e^{\frac{\eta}{\varepsilon}(s-t)}\left(1+\hat{\mathbb{E}}\left[\left|\widetilde{S}_{s}^{\varepsilon, x}\right|^{2}\right]\right) d s, \forall t \in[0, T] .
$$

Following the proof of Assertion (iii) in Lemma 4.1, we could get

$$
\hat{\mathbb{E}}\left[\sup _{0 \leq t \leq T}\left|\widetilde{S}_{t}^{\varepsilon, x}\right|^{2}\right]+\sup _{0 \leq t \leq T} \hat{\mathbb{E}}\left[\left|\bar{S}_{t}^{\varepsilon, x}\right|^{2}\right] \leq C\left(L_{1}, \eta, T\right)\left(1+|\tilde{x}|^{2}+|\bar{x}|^{2}\right) .
$$

On the other hand, if $\varepsilon>\frac{\eta^{2}}{C\left(L_{1}\right)}$, then all Lipschitz constants are uniformly bounded, and the result is trivial.

Step 2 (Assertion (ii)). Applying $G$-Itô's formula yields that

$$
\begin{aligned}
& e^{\frac{\eta}{\varepsilon} t}\left|\bar{S}_{t}^{\varepsilon, x}-\bar{X}_{t}^{\varepsilon, x}\right|^{2}-M_{t}^{2}-\frac{\eta}{\varepsilon} \int_{0}^{t} e^{\frac{\eta}{\varepsilon} s}\left|\bar{S}_{s}^{\varepsilon, x}-\bar{X}_{s}^{\varepsilon, x}\right|^{2} d s \\
& \leq \frac{2}{\varepsilon} \int_{0}^{t} e^{\frac{\eta}{\varepsilon} s}\left[\left\langle\bar{S}_{s}^{\varepsilon, x}-\bar{X}_{s}^{\varepsilon, x}, \bar{b}\left(\widetilde{S}_{s}^{\varepsilon, x}, \bar{S}_{s}^{\varepsilon, x}\right)-\bar{b}\left(\widetilde{X}_{s}^{\varepsilon, x}, \bar{X}_{s}^{\varepsilon, x}\right)\right\rangle+G\left(\Gamma_{s}^{\top} \Gamma_{s}\right)\right] d s \\
& \quad+\frac{2}{\sqrt{\varepsilon}} \int_{0}^{t} e^{\frac{\eta}{\varepsilon} s}\left[\left\langle\bar{S}_{s}^{\varepsilon, x}-\bar{X}_{s}^{\varepsilon, x}, \bar{b}^{1}\left(\widetilde{S}_{s}^{\varepsilon, x}, \bar{S}_{s}^{\varepsilon, x}\right)\right\rangle+G\left(\Gamma_{s}^{\top} \bar{\sigma}^{1}\left(\widetilde{S}_{s}^{\varepsilon, x}, \bar{S}_{s}^{\varepsilon, x}\right)+\left(\bar{\sigma}^{1}\left(\widetilde{S}_{s}^{\varepsilon, x}, \bar{S}_{s}^{\varepsilon, x}\right)\right)^{\top} \Gamma_{s}\right)\right] d s \\
& \quad+2 \int_{0}^{t} e^{\frac{\eta}{\varepsilon} s}\left[\left\langle\bar{S}_{s}^{\varepsilon, x}-\bar{X}_{s}^{\varepsilon, x}, \bar{b}^{2}\left(\widetilde{S}_{s}^{\varepsilon, x}, \bar{S}_{s}^{\varepsilon, x}\right)\right\rangle+G\left(\left(\bar{\sigma}^{1}\left(\widetilde{S}_{s}^{\varepsilon, x}, \bar{S}_{s}^{\varepsilon, x}\right)\right)^{\top} \bar{\sigma}^{1}\left(\widetilde{S}_{s}^{\varepsilon, x}, \bar{S}_{s}^{\varepsilon, x}\right)\right)\right] d s,
\end{aligned}
$$

where $M_{t}^{2}$ is a symmetric $G$-martingale and $\Gamma_{t}=\bar{\sigma}\left(\widetilde{S}_{t}^{\varepsilon, x}, \bar{S}_{t}^{\varepsilon, x}\right)-\bar{\sigma}\left(\widetilde{X}_{t}^{\varepsilon, x}, \bar{X}_{t}^{\varepsilon, x}\right)$.

According to assumptions (H1) and (H2), we derive that

$$
\begin{aligned}
& 2\left\langle\bar{S}_{s}^{\varepsilon, x}-\bar{X}_{s}^{\varepsilon, x}, \bar{b}\left(\widetilde{S}_{s}^{\varepsilon, x}, \bar{S}_{s}^{\varepsilon, x}\right)-\bar{b}\left(\widetilde{X}_{s}^{\varepsilon, x}, \bar{X}_{s}^{\varepsilon, x}\right)\right\rangle+2 G\left(\Gamma_{s}^{\top} \Gamma_{s}\right) \\
& \leq-\frac{3}{2} \eta\left|\bar{S}_{s}^{\varepsilon, x}-\bar{X}_{s}^{\varepsilon, x}\right|^{2}+C\left(L_{1}, \eta\right)\left|\widetilde{S}_{s}^{\varepsilon, x}-\widetilde{X}_{s}^{\varepsilon, x}\right|^{2},
\end{aligned}
$$


and

$$
\begin{aligned}
& 2\left\langle\bar{S}_{s}^{\varepsilon, x}-\bar{X}_{s}^{\varepsilon, x}, \bar{b}^{1}\left(\widetilde{S}_{s}^{\varepsilon, x}, \bar{S}_{s}^{\varepsilon, x}\right)\right\rangle+2 G\left(\Gamma_{s}^{\top} \bar{\sigma}^{1}\left(\widetilde{S}_{s}^{\varepsilon, x}, \bar{S}_{s}^{\varepsilon, x}\right)+\left(\bar{\sigma}^{1}\left(\widetilde{S}_{s}^{\varepsilon, x}, \bar{S}_{s}^{\varepsilon, x}\right)\right)^{\top} \Gamma_{s}\right) \\
& \leq \frac{\eta}{4 \sqrt{\varepsilon}}\left|\bar{S}_{s}^{\varepsilon, x}-\bar{X}_{s}^{\varepsilon, x}\right|^{2}+\frac{\eta}{4 \sqrt{\varepsilon}}\left|\widetilde{S}_{s}^{\varepsilon, x}-\widetilde{X}_{s}^{\varepsilon, x}\right|^{2}+C\left(L_{1}, \eta\right) \sqrt{\varepsilon}\left(1+\left|\widetilde{S}_{s}^{\varepsilon, x}\right|^{2}+\left|\bar{S}_{s}^{\varepsilon, x}\right|^{2}\right), \\
& 2\left\langle\bar{S}_{s}^{\varepsilon, x}-\bar{X}_{s}^{\varepsilon, x}, \bar{b}^{2}\left(\widetilde{S}_{s}^{\varepsilon, x}, \bar{S}_{s}^{\varepsilon, x}\right)\right\rangle+2 G\left(\left(\bar{\sigma}^{1}\left(\widetilde{S}_{s}^{\varepsilon, x}, \bar{S}_{s}^{\varepsilon, x}\right)\right)^{\top} \bar{\sigma}^{1}\left(\widetilde{S}_{s}^{\varepsilon, x}, \bar{S}_{s}^{\varepsilon, x}\right)\right) \\
& \leq \frac{\eta}{4 \varepsilon}\left|\bar{S}_{s}^{\varepsilon, x}-\bar{X}_{s}^{\varepsilon, x}\right|^{2}+C\left(L_{1}, \eta\right)\left(1+\left|\widetilde{S}_{s}^{\varepsilon, x}\right|^{2}+\left|\bar{S}_{s}^{\varepsilon, x}\right|^{2}\right) .
\end{aligned}
$$

Putting the above four inequalities together, we get that

$$
\begin{aligned}
e^{\frac{\eta}{\varepsilon} t}\left|\bar{S}_{t}^{\varepsilon, x}-\bar{X}_{t}^{\varepsilon, x}\right|^{2} \leq & M_{t}^{2}+\frac{C\left(L_{1}, \eta\right)}{\varepsilon} \int_{0}^{t} e^{\frac{\eta}{\varepsilon} s}\left|\widetilde{S}_{s}^{\varepsilon, x}-\widetilde{X}_{s}^{\varepsilon, x}\right|^{2} d s \\
& +C\left(L_{1}, \eta\right) \int_{0}^{t} e^{\frac{\eta}{\varepsilon} s}\left(1+\left|\widetilde{S}_{s}^{\varepsilon, x}\right|^{2}+\left|\bar{S}_{s}^{\varepsilon, x}\right|^{2}\right) d s
\end{aligned}
$$

Taking $G$-expectation on both sides and recalling Assertion (i), we obtain that for each $t \in[0, T]$,

$$
\hat{\mathbb{E}}\left[\left|\bar{S}_{t}^{\varepsilon, x}-\bar{X}_{t}^{\varepsilon, x}\right|^{2}\right] \leq C\left(L_{1}, \eta, T\right)\left(1+|x|^{2}\right) \varepsilon+\frac{C\left(L_{1}, \eta\right)}{\varepsilon} \int_{0}^{t} e^{\frac{\eta}{\varepsilon}(s-t)} \hat{\mathbb{E}}\left[\left|\widetilde{S}_{s}^{\varepsilon, x}-\widetilde{X}_{s}^{\varepsilon, x}\right|^{2}\right] d s,
$$

which indicates that

$$
\int_{0}^{t} \hat{\mathbb{E}}\left[\left|\bar{S}_{s}^{\varepsilon, x}-\bar{X}_{s}^{\varepsilon, x}\right|^{2}\right] d s \leq C\left(L_{1}, \eta, T\right)\left(1+|x|^{2}\right) \varepsilon+C\left(L_{1}, \eta\right) \int_{0}^{t} \hat{\mathbb{E}}\left[\sup _{0 \leq r \leq s}\left|\widetilde{S}_{r}^{\varepsilon, x}-\widetilde{X}_{r}^{\varepsilon, x}\right|^{2}\right] d s .
$$

It follows from Hölder's inequality and BDG's inequality that

$$
\hat{\mathbb{E}}\left[\sup _{0 \leq s \leq t}\left|\widetilde{S}_{s}^{\varepsilon, x}-\widetilde{X}_{s}^{\varepsilon, x}\right|^{2}\right] \leq C\left(L_{1}, \eta, T\right)\left(\left(1+|x|^{2}\right) \varepsilon+\int_{0}^{t} \hat{\mathbb{E}}\left[\sup _{0 \leq r \leq s}\left|\widetilde{S}_{r}^{\varepsilon, x}-\widetilde{X}_{r}^{\varepsilon, x}\right|^{2}\right] d s\right),
$$

which together with Gronwall's inequality implies that

$$
\hat{\mathbb{E}}\left[\sup _{0 \leq s \leq T}\left|\widetilde{S}_{s}^{\varepsilon, x}-\widetilde{X}_{s}^{\varepsilon, x}\right|^{2}\right] \leq C\left(L_{1}, \eta, T\right)\left(1+|x|^{2}\right) \varepsilon
$$

The proof is complete.

Then we have the following asymptotics result.

Theorem 5.2 Suppose assumptions (H1)-(H4) hold. Then, for each $\varphi \in C\left(\mathbb{R}^{n}\right)$ of polynomial growth,

$$
\lim _{\varepsilon \rightarrow 0} \hat{\mathbb{E}}\left[\varphi\left(\widetilde{S}_{t}^{\varepsilon, x}\right)\right]=\widetilde{u}(t, \tilde{x}), \forall(t, \tilde{x}, \bar{x}) \in[0, \infty) \times \mathbb{R}^{2 n},
$$

where $\widetilde{u}$ is the unique viscosity solution to the averaged PDE (91) satisfying the polynomial growth condition.

Proof. It suffices to prove that $\lim _{\varepsilon \rightarrow 0} \hat{\mathbb{E}}\left[\left|\varphi\left(\widetilde{S}_{t}^{\varepsilon, x}\right)-\varphi\left(\widetilde{X}_{t}^{\varepsilon, x}\right)\right|\right]=0$. Note that $\varphi$ satisfies the polynomial growth condition. According to Lemma 4.3, we obtain that for each $N>0$,

$$
\hat{\mathbb{E}}\left[\left|\varphi\left(\widetilde{S}_{t}^{\varepsilon, x}\right)-\varphi\left(\widetilde{X}_{t}^{\varepsilon, x}\right)\right|\right] \leq \hat{\mathbb{E}}\left[\left|\varphi\left(\widetilde{S}_{t}^{\varepsilon, x}\right)-\varphi\left(\widetilde{X}_{t}^{\varepsilon, x}\right)\right| I_{\left|\widetilde{S}_{t}^{\varepsilon, x}\right| \leq N} I_{\left|\widetilde{X}_{t}^{\varepsilon, x}\right| \leq N}\right]+C\left(L_{1}, L_{2}, T, \varphi\right) \frac{1+|\tilde{x}|^{C(\varphi)}}{N} .
$$


For each $\epsilon>0$, there is a constant $\delta>0$ such that $|\varphi(x)-\varphi(y)| \leq \epsilon$ whenever $|x| \leq N,|y| \leq N$, and $|x-y| \leq \delta$. It follows that

$$
\begin{aligned}
& \hat{\mathbb{E}}\left[\left|\varphi\left(\widetilde{S}_{t}^{\varepsilon, x}\right)-\varphi\left(\widetilde{X}_{t}^{\varepsilon, x}\right)\right| I_{\left|\widetilde{S}_{t}^{\varepsilon, x}\right| \leq N} I_{\left|\widetilde{X}_{t}^{\varepsilon, x}\right| \leq N}\right] \leq \epsilon+\hat{\mathbb{E}}\left[\left|\varphi\left(\widetilde{S}_{t}^{\varepsilon, x}\right)-\varphi\left(\widetilde{X}_{t}^{\varepsilon, x}\right)\right| I_{\left|\widetilde{S}_{t}^{\varepsilon, x}-\widetilde{X}_{t}^{\varepsilon, x}\right| \geq \delta}\right] \\
& \leq \epsilon+\hat{\mathbb{E}}\left[\left|\varphi\left(\widetilde{S}_{t}^{\varepsilon, x}\right)-\varphi\left(\widetilde{X}_{t}^{\varepsilon, x}\right)\right|^{2}\right] \hat{\mathbb{E}}\left[\left|\widetilde{S}_{t}^{\varepsilon, x}-\widetilde{X}_{t}^{\varepsilon, x}\right|^{2}\right] \delta^{-2} \leq \epsilon+C\left(L_{1}, L_{2}, \eta, T, \varphi\right)\left(1+|x|^{C(\varphi)}\right) \frac{\varepsilon}{\delta^{2}}
\end{aligned}
$$

where we have used Lemma 5.1 in the last inequality. As a result, we deduce that

$$
\limsup _{\varepsilon \rightarrow 0} \hat{\mathbb{E}}\left[\left|\varphi\left(\widetilde{S}_{t}^{\varepsilon, x}\right)-\varphi\left(\widetilde{X}_{t}^{\varepsilon, x}\right)\right|\right] \leq \epsilon+C(\varphi) \frac{1+|\tilde{x}|^{C(\varphi)}}{N} .
$$

Sending $\epsilon \rightarrow 0$, and then letting $N \rightarrow \infty$, we could complete the proof.

\section{Acknowledgements}

The authors would like to thank the editor and the anonymous referees for their valuable suggestions and comments which led to a much improved version of the manuscript.

\section{Appendix A: Ergodic theory in the $G$-expectation framework}

In what follows, we shall recall some basic results about ergodic $G$-BSDE: for each $x \in \mathbb{R}^{n}$ and for any $0 \leq t \leq T<\infty$,

$$
\left\{\begin{array}{l}
X_{t}^{x}=x+\int_{0}^{t} b\left(X_{s}^{x}\right) d s+\sum_{i, j=1}^{d} \int_{0}^{t} h_{i j}\left(X_{s}^{x}\right) d\left\langle B^{i}, B^{j}\right\rangle_{s}+\int_{0}^{t} \sigma\left(X_{s}^{x}\right) d B_{s}, \\
Y_{t}^{x}=Y_{T}^{x}+\int_{t}^{T}\left(g\left(X_{s}^{x}\right)-\lambda\right) d s+\sum_{i, j=1}^{d} \int_{t}^{T} g_{i j}^{\prime}\left(X_{s}^{x}\right) d\left\langle B^{i}, B^{j}\right\rangle_{s}-\int_{t}^{T} Z_{s}^{x} d B_{s}-\left(K_{T}^{x}-K_{t}^{x}\right),
\end{array}\right.
$$

where $b, h_{i j}=h_{j i}: \mathbb{R}^{n} \rightarrow \mathbb{R}^{n}, \sigma: \mathbb{R}^{n} \rightarrow \mathbb{R}^{n \times d}$ and $g, g_{i j}^{\prime}: \mathbb{R}^{n} \rightarrow \mathbb{R}$ are deterministic functions satisfying the following conditions.

(A1) There exist two constant $\kappa_{1}>0$ and $\kappa_{2}>0$ such that for each $x, x^{\prime} \in \mathbb{R}^{n}$,

$$
\left|\ell(x)-\ell\left(x^{\prime}\right)\right| \leq \kappa_{1}\left|x-x^{\prime}\right|, \text { for } \ell=b, h_{i j}, \sigma, \text { and }\left|\ell(x)-\ell\left(x^{\prime}\right)\right| \leq \kappa_{2}\left|x-x^{\prime}\right|, \text { for } \ell=g, g^{\prime} .
$$

(A2) There exists a constant $\eta>0$ such that for each $x, x^{\prime} \in \mathbb{R}^{n}$,

$$
\begin{aligned}
& G\left(\left(\sigma(x)-\sigma\left(x^{\prime}\right)\right)^{\top}\left(\sigma(x)-\sigma\left(x^{\prime}\right)\right)+2\left[\left\langle x-x^{\prime}, h_{i j}(x)-h_{i j}\left(x^{\prime}\right)\right\rangle\right]_{i, j=1}^{d}\right)+\left\langle x-x^{\prime}, b(x)-b\left(x^{\prime}\right)\right\rangle \\
& \leq-\eta\left|x-x^{\prime}\right|^{2} .
\end{aligned}
$$

Lemma A.1 Under assumptions (A1) and (A2), there exists a constant $C\left(\kappa_{1}, \eta\right)$, such that for any $x, x^{\prime} \in \mathbb{R}^{n}$ and $t, s \geq 0$,

(i) $\hat{\mathbb{E}}\left[\left|X_{t}^{x}\right|^{2}\right] \leq C\left(\kappa_{1}, \eta\right)\left(1+|x|^{2}+|\bar{\kappa}|^{2}\right)$,

(ii) $\hat{\mathbb{E}}\left[\left|X_{t}^{x}-X_{t}^{x^{\prime}}\right|^{2}\right] \leq \exp (-2 \eta t)\left|x-x^{\prime}\right|^{2}$, 
where $\bar{\kappa}:=\max \left\{|b(0)|,|\sigma(0)|,\left|h_{i j}(0)\right|, 1 \leq i, j \leq d\right\}$.

Proof. The proof is immediate from Lemma 3.2 of 23 or Lemma 4.1 of 26 .

Let $S_{G}^{0}(0, T)=\left\{h\left(t, B_{t_{1} \wedge t}, \cdots, B_{t_{n} \wedge t}\right): t_{1}, \ldots, t_{n} \in[0, T], h \in C_{b . l i p}\left(\mathbb{R}^{n \times d+1}\right)\right\}$. For $\eta \in S_{G}^{0}(0, T)$, set $\|\eta\|_{S_{G}^{2}}=\left\{\hat{\mathbb{E}}\left[\sup _{t \in[0, T]}\left|\eta_{t}\right|^{2}\right]\right\}^{\frac{1}{2}}$. Denote by $S_{G}^{2}(0, T)$ the completion of $S_{G}^{0}(0, T)$ under the norm $\|\cdot\|_{S_{G}^{2}}$ (see [32] for more related research). For the sake of brevity, denote by $\mathfrak{S}_{G}^{2}(0, \infty)$ the collection of processes $\left(Y_{t}, Z_{t}, K_{t}\right)_{t \geq 0}$ such that, for each $T>0,\left(Y_{t}\right)_{t \in[0, T]} \in S_{G}^{2}(0, T),\left(Z_{t}\right)_{t \in[0, T]} \in M_{G}^{2}\left(0, T ; \mathbb{R}^{d}\right)$ and $\left(K_{t}\right)_{t \in[0, T]} \in S_{G}^{2}(0, T)$ is a continuous non-increasing $G$-martingale starting from origin.

Lemma A.2 Suppose that (A1) and (A2) hold. Then, the $G$-EBSDE (28) has a solution $\left(Y^{x}, Z^{x}, K^{x}, \lambda\right) \in$ $\mathfrak{S}_{G}^{2}(0, \infty) \times \mathbb{R}$ for each $x \in \mathbb{R}^{n}$, where the constant $\lambda$ is independent of the argument $x$. Moreover, there exists a continuous function $v$ satisfying

$$
v(0)=0,\left|v(x)-v\left(x^{\prime}\right)\right| \leq C(\eta) \kappa_{2}\left|x-x^{\prime}\right|, \forall x, x^{\prime} \in \mathbb{R}^{n},
$$

such that $Y_{t}^{x}=v\left(X_{t}^{x}\right)$ for each $(t, x) \in[0, \infty) \times \mathbb{R}^{n}$.

Proof. We shall give the sketch of the proof for readers' convenience. Without loss of generality, assume $g_{i j}^{\prime}=0$. For each $\epsilon>0$, consider the following $G$-BSDE with infinite horizon:

$$
Y_{t}^{\epsilon, x}=Y_{T}^{\epsilon, x}+\int_{t}^{T}\left(g\left(X_{s}^{x}\right)-\epsilon Y_{s}^{\epsilon, x}\right) d s-\int_{t}^{T} Z_{s}^{\epsilon, x} d B_{s}-\left(K_{T}^{\epsilon, x}-K_{t}^{\epsilon, x}\right), \quad \forall 0 \leq t \leq T<\infty .
$$

We define the function $v^{\epsilon}(x):=Y_{0}^{\epsilon, x}$ for each $x \in \mathbb{R}^{n}$. Then from Lemma A.1, the proof of Theorem 3.1 and Lemma 4.2 of [26], we have that

$$
\left|v^{\epsilon}(x)\right| \leq C\left(\kappa_{1}, \kappa_{2}, \eta\right) \frac{1+|x|+|\bar{\kappa}|}{\epsilon} \text { and }\left|v^{\epsilon}(x)-v^{\epsilon}\left(x^{\prime}\right)\right| \leq C(\eta) \kappa_{2}\left|x-x^{\prime}\right| .
$$

Denote $\bar{v}^{\epsilon}(x)=v^{\epsilon}(x)-v^{\epsilon}(0)$. Note that $\bar{v}^{\epsilon}(x)$ is a uniformly Lipschitz function. Thus, by a diagonal procedure, we can construct a sequence $\epsilon_{m} \downarrow 0$ such that $\bar{v}^{\epsilon_{m}}(x) \rightarrow v(x)$ for all $x \in \mathbb{R}^{n}$ and $\epsilon_{m} v^{\epsilon_{m}}(0) \rightarrow$ $\lambda$ for some real number $\lambda$. Finally, by a similar analysis as in Theorem 5.1 of [26], we can get the desired result.

Then, we have the following asymptotic property, which can be seen as the ergodic theorem in the $G$-expectation framework.

Lemma A.3 Assume conditions (A1) and (A2) hold. Then, for each $T \in[0, \infty)$, we have

$$
\left|\hat{\mathbb{E}}\left[\int_{0}^{T} g\left(X_{s}^{x}\right) d s+\sum_{i, j=1}^{d} \int_{0}^{T} g_{i j}^{\prime}\left(X_{s}^{x}\right) d\left\langle B^{i}, B^{j}\right\rangle_{s}\right]-\lambda T\right| \leq C\left(\kappa_{1}, \eta\right) \kappa_{2}(1+|x|+|\bar{\kappa}|), \forall x \in \mathbb{R}^{n} .
$$

where $\bar{\kappa}$ and $\lambda$ are given by Lemma A.1 and Lemma A.2, respectively. In particular,

$$
\lambda=\lim _{T \rightarrow \infty} \frac{1}{T} \hat{\mathbb{E}}\left[\int_{0}^{T} g\left(X_{s}^{x}\right) d s+\sum_{i, j=1}^{d} \int_{0}^{T} g_{i j}^{\prime}\left(X_{s}^{x}\right) d\left\langle B^{i}, B^{j}\right\rangle_{s}\right] .
$$

Proof. From equation (28), we get that

$$
Y_{0}^{x}=\hat{\mathbb{E}}\left[Y_{T}^{x}+\int_{0}^{T} g\left(X_{s}^{x}\right) d s+\sum_{i, j=1}^{d} \int_{0}^{T} g_{i j}^{\prime}\left(X_{s}^{x}\right) d\left\langle B^{i}, B^{j}\right\rangle_{s}-\lambda T\right],
$$


which implies that

$$
\begin{aligned}
& \left|\hat{\mathbb{E}}\left[\int_{0}^{T} g\left(X_{s}^{x}\right) d s+\sum_{i, j=1}^{d} \int_{0}^{T} g_{i j}^{\prime}\left(X_{s}^{x}\right) d\left\langle B^{i}, B^{j}\right\rangle_{s}\right]-\lambda T\right| \\
& \leq\left|\hat{\mathbb{E}}\left[Y_{T}^{x}+\int_{0}^{T} g\left(X_{s}^{x}\right) d s+\sum_{i, j=1}^{d} \int_{0}^{T} g_{i j}^{\prime}\left(X_{s}^{x}\right) d\left\langle B^{i}, B^{j}\right\rangle_{s}\right]-\lambda T\right|+\hat{\mathbb{E}}\left[\left|Y_{T}^{x}\right|\right] \leq\left|Y_{0}^{x}\right|+\hat{\mathbb{E}}\left[\left|Y_{T}^{x}\right|\right] .
\end{aligned}
$$

Recalling Lemma A.2, there exists a constant $C(\eta)$ such that

$$
\left|Y_{s}^{x}\right| \leq C(\eta) \kappa_{2}\left|X_{s}^{x}\right|, \quad \forall s \geq 0,
$$

which together with Assertion (i) of Lemma A.1, indicates that

$$
\hat{\mathbb{E}}\left[\left|Y_{T}^{x}\right|\right] \leq C(\eta) \kappa_{2} \hat{\mathbb{E}}\left[\left|X_{T}^{x}\right|\right] \leq C\left(\kappa_{1}, \eta\right) \kappa_{2}(1+|x|+|\bar{\kappa}|)
$$

It follows that

$$
\left|\hat{\mathbb{E}}\left[\int_{0}^{T} g\left(X_{s}^{x}\right) d s+\sum_{i, j=1}^{d} \int_{0}^{T} g_{i j}^{\prime}\left(X_{s}^{x}\right) d\left\langle B^{i}, B^{j}\right\rangle_{s}\right]-\lambda T\right| \leq C\left(\kappa_{1}, \eta\right) \kappa_{2}(1+|x|+|\bar{\kappa}|),
$$

which completes the proof.

\section{Appendix B: Comparison theorem for the averaged PDE}

In this appendix, we shall state the comparison theorem for PDE (9).

Lemma B.1 Let $\widetilde{v}^{1}$ be a viscosity subsolution and $\widetilde{v}^{2}$ be a viscosity supersolution to PDE (9) satisfying the polynomial growth condition, respectively. Then $\widetilde{v}^{1} \leq \widetilde{v}^{2}$ on $[0, T] \times \mathbb{R}^{n}$ provided that $\left.\widetilde{v}^{1}\right|_{t=0} \leq$ $\left.\widetilde{v}^{2}\right|_{t=0}$.

Proof. The main idea is from Theorem 8.6 in [14] and Theorem 2.2 in Appendix C of [38]. For reader's convenience, we shall give the sketch of the proof.

For some constant $\lambda>0$ to be chosen below, we set $\xi(\tilde{x}):=\left(1+|\tilde{x}|^{2}\right)^{l / 2}$ and

$$
\widetilde{v}_{1}(t, \tilde{x}):=\widetilde{v}^{1}(t, \tilde{x}) \xi^{-1}(\tilde{x}) e^{-\lambda t}, \widetilde{v}_{2}(t, \tilde{x}):=-\widetilde{v}^{2}(t, \tilde{x}) \xi^{-1}(\tilde{x}) e^{-\lambda t},
$$

where $l \geq 2$ is chosen to be large enough such that $\left|\widetilde{v}_{i}\right| \rightarrow 0$ uniformly as $x \rightarrow \infty$. It is easy to check that, $\widetilde{v}_{i}$ is a bounded viscosity subsolution of

$$
\partial_{t} \widetilde{v}_{i}+\lambda \widetilde{v}_{i}-\widetilde{G}_{i}^{*}\left(\tilde{x}, \widetilde{v}_{i}, D \widetilde{v}_{i}, D^{2} \widetilde{v}_{i}\right)=0,
$$

where the function $\widetilde{G}_{1}^{*}(\tilde{x}, v, p, X)=\widetilde{G}^{*}(\tilde{x}, v, p, X), \widetilde{G}_{2}^{*}(\tilde{x}, v, p, X)=-\widetilde{G}^{*}(\tilde{x},-v,-p,-X)$ and

$$
\begin{aligned}
\widetilde{G}^{*}(\tilde{x}, v, p, X): & =e^{-\lambda t} \xi^{-1} \widetilde{G}\left(\tilde{x}, e^{\lambda t}(p \xi(\tilde{x})+v D \xi(\tilde{x})), e^{\lambda t}\left(X \xi(\tilde{x})+p \otimes D \xi(\tilde{x})+D \xi(\tilde{x}) \otimes p+v D^{2} \xi(\tilde{x})\right)\right) \\
& =\widetilde{G}(\tilde{x}, p+v \eta(\tilde{x}), X+p \otimes \eta(\tilde{x})+\eta(\tilde{x}) \otimes p+v \kappa(\tilde{x}))
\end{aligned}
$$

for any $(\tilde{x}, v, p, X) \in \mathbb{R}^{n} \times \mathbb{R} \times \mathbb{R}^{n} \times \mathbb{S}(n)$. Here $p \otimes \eta(\tilde{x})=\left[p^{i} \eta^{j}(\tilde{x})\right]_{i, j}$ and

$$
\begin{aligned}
& \eta(\tilde{x}):=\xi^{-1}(\tilde{x}) D \xi(\tilde{x})=l\left(1+|\tilde{x}|^{2}\right)^{-1} \tilde{x}, \\
& \kappa(\tilde{x}):=\xi^{-1}(\tilde{x}) D^{2} \xi(\tilde{x})=l\left(1+|\tilde{x}|^{2}\right)^{-1} I_{n}+l(l-2)\left(1+|\tilde{x}|^{2}\right)^{-2} \tilde{x} \otimes \tilde{x} .
\end{aligned}
$$


Note that $l \geq 2, \eta$ and $\kappa$ are uniformly bounded functions. Then, using Assertion (iv) of Lemma 3.3. we could choose $\lambda$ large enough, so that the function

$$
v \rightarrow-\lambda v+\widetilde{G}^{*}(\tilde{x}, v, p, X) \text { is non-increasing for any }(\tilde{x}, p, X) \in \mathbb{R}^{n} \times \mathbb{R}^{n} \times \mathbb{S}(n) .
$$

Next, we shall verify that $\widetilde{G}^{*}$ satisfies the regularity condition (3.14) in [9] for the comparison principle. Suppose that $A, B \in \mathbb{S}(n)$ satisfies

$$
\left(\begin{array}{cc}
A & 0 \\
0 & B
\end{array}\right) \leq 3 \alpha\left(\begin{array}{cc}
I_{n} & -I_{n} \\
-I_{n} & I_{n}
\end{array}\right)
$$

for some $\alpha>0$. Note that $G(A) \leq \frac{1}{2} \bar{\sigma}^{2} \operatorname{tr}[A]$ for any $A \geq 0$. Then, we get that

$$
\begin{aligned}
& \hat{\mathbb{E}}\left[\sum_{i, j=1}^{d} \int_{0}^{T} \widetilde{\sigma}_{i j}^{A}\left(\tilde{x}, \bar{X}_{s}^{(\tilde{x}, \bar{x})}\right) d\left\langle B^{i}, B^{j}\right\rangle_{s}-\sum_{i, j=1}^{d} \int_{0}^{T} \widetilde{\sigma}_{i j}^{-B}\left(\tilde{y}, \bar{X}_{s}^{(\tilde{y}, \bar{x})}\right) d\left\langle B^{i}, B^{j}\right\rangle_{s}\right] \\
& \leq 2 \hat{\mathbb{E}}\left[\int_{0}^{T} G\left(\tilde{\sigma}^{\top}\left(\tilde{x}, \bar{X}_{s}^{(\tilde{x}, \bar{x})}\right) A \widetilde{\sigma}\left(\tilde{x}, \bar{X}_{s}^{(\tilde{x}, \bar{x})}\right)+\tilde{\sigma}^{\top}\left(\tilde{y}, \bar{X}_{s}^{(\tilde{y}, \bar{x})}\right) B \tilde{\sigma}\left(\tilde{y}, \bar{X}_{s}^{(\tilde{y}, \bar{x})}\right)\right) d s\right] \\
& \leq 3 \bar{\sigma}^{2} \alpha \hat{\mathbb{E}}\left[\int_{0}^{T}\left|\tilde{\sigma}\left(\tilde{x}, \bar{X}_{s}^{(\tilde{x}, \bar{x})}\right)-\tilde{\sigma}\left(\tilde{y}, \bar{X}_{s}^{(\tilde{y}, \bar{x})}\right)\right|^{2} d s\right] \leq C\left(L_{1}, \eta\right) \alpha|\tilde{x}-\tilde{y}|^{2} T,
\end{aligned}
$$

where we have used assumption (H1) and estimate (8) in the last inequality. Thus, from the definitions of $\widetilde{G}, \widetilde{G}^{*}$ and by a similar analysis as the proof of Assertion (iv) of Lemma 3.3 , we conclude that

$$
\widetilde{G}^{*}(\tilde{x}, v, \alpha(\tilde{x}-\tilde{y}), A)-\widetilde{G}^{*}(\tilde{y}, v, \alpha(\tilde{x}-\tilde{y}),-B) \leq C\left(L_{1}, L_{2}, \eta\right)\left(1+|\tilde{x}|^{2}+|\tilde{y}|^{2}\right)\left(|v||\tilde{x}-\tilde{y}|+\alpha|\tilde{x}-\tilde{y}|^{2}\right) .
$$

Finally, we will prove $\widetilde{v}_{1}+\widetilde{v}_{2} \leq 0$. By the proof of Theorem 2.2 in [38], it suffices to prove the result under the additional assumptions: for each $\bar{\delta}>0$,

$$
\partial_{t} \widetilde{v}_{i}+\lambda \widetilde{v}_{i}-\widetilde{G}_{i}^{*}\left(\tilde{x}, \widetilde{v}_{i}, D \widetilde{v}_{i}, D^{2} \widetilde{v}_{i}\right) \leq-\bar{\delta} / T^{2} \text {, and } \lim _{t \rightarrow T} \widetilde{v}_{i}(t, \tilde{x})=-\infty \text { uniformly on } \mathbb{R}^{n} \text {. }
$$

Assume the contrary that

$$
\sup _{(t, x) \in[0, T) \times \mathbb{R}^{n}}\left(\widetilde{v}_{1}(t, x)+\widetilde{v}_{2}(t, x)\right)>0 .
$$

Note that $\left(\widetilde{v}_{1}(t, \tilde{x})\right)^{+}+\left(\widetilde{v}_{2}(t, \tilde{x})\right)^{+} \rightarrow 0$ uniformly as $\tilde{x} \rightarrow \infty$. Thus, taking $\beta_{1}=\beta_{2}=1$ and following the proof of Theorem 2.2 in [38] line by line, for large enough $\alpha>0$, we could find some point $\left(t^{\alpha}, \tilde{x}_{1}^{\alpha}, \tilde{x}_{2}^{\alpha}\right)$ inside a compact subset of $[0, T) \times \mathbb{R}^{2 n}$, so that $\widetilde{v}_{1}\left(t^{\alpha}, \tilde{x}_{1}^{\alpha}\right)+\widetilde{v}_{2}\left(t^{\alpha}, \tilde{x}_{2}^{\alpha}\right)-\frac{\alpha}{2}\left|\tilde{x}_{2}^{\alpha}-\tilde{x}_{1}^{\alpha}\right|^{2}>0$ and

$$
\lim _{\alpha \rightarrow \infty} \alpha\left|\tilde{x}_{1}^{\alpha}-\tilde{x}_{2}^{\alpha}\right|^{2}=0 \text {, and } \lim _{\alpha \rightarrow \infty}\left(t^{\alpha}, \tilde{x}_{1}^{\alpha}, \tilde{x}_{2}^{\alpha}\right)=\left(t^{*}, \tilde{x}^{*}, \tilde{x}^{*}\right) \text { for some }\left(t^{*}, \tilde{x}^{*}\right) \in(0, T) \times \mathbb{R}^{n} .
$$

Then, there exist $b_{i}^{\alpha} \in \mathbb{R}, X_{i}^{\alpha} \in \mathbb{S}(n)$ such that $b_{1}^{\alpha}+b_{2}^{\alpha}=0$,

$$
\left(b_{1}^{\alpha}, \alpha\left(\tilde{x}_{1}^{\alpha}-\tilde{x}_{2}^{\alpha}\right), X_{1}^{\alpha}\right) \in \overline{\mathcal{P}}^{2,+} \widetilde{v}_{1}\left(t^{\alpha}, \tilde{x}_{1}^{\alpha}\right), \quad\left(b_{2}^{\alpha}, \alpha\left(\tilde{x}_{2}^{\alpha}-\tilde{x}_{1}^{\alpha}\right), X_{2}^{\alpha}\right) \in \overline{\mathcal{P}}^{2,+} \widetilde{v}_{2}\left(t^{\alpha}, \tilde{x}_{2}^{\alpha}\right),
$$

and

$$
\left(\begin{array}{cc}
X_{1}^{\alpha} & 0 \\
0 & X_{2}^{\alpha}
\end{array}\right) \leq 3 \alpha\left(\begin{array}{cc}
I_{n} & -I_{n} \\
-I_{n} & I_{n}
\end{array}\right)
$$


Moreover, it follows from equation (32) that

$$
\begin{aligned}
& b_{1}^{\alpha}+\lambda \widetilde{v}_{1}\left(t^{\alpha}, \tilde{x}_{1}^{\alpha}\right)-\widetilde{G}_{1}^{*}\left(\tilde{x}_{1}^{\alpha}, \widetilde{v}_{1}\left(t^{\alpha}, \tilde{x}_{1}^{\alpha}\right), \alpha\left(\tilde{x}_{1}^{\alpha}-\tilde{x}_{2}^{\alpha}\right), X_{1}^{\alpha}\right) \leq-\bar{\delta} / T^{2}, \\
& b_{2}^{\alpha}+\lambda \widetilde{v}_{2}\left(t^{\alpha}, \tilde{x}_{2}^{\alpha}\right)-\widetilde{G}_{2}^{*}\left(\tilde{x}_{2}^{\alpha}, \widetilde{v}_{2}\left(t^{\alpha}, \tilde{x}_{2}^{\alpha}\right), \alpha\left(\tilde{x}_{2}^{\alpha}-\tilde{x}_{1}^{\alpha}\right), X_{2}^{\alpha}\right) \leq-\bar{\delta} / T^{2} .
\end{aligned}
$$

According to the definition of $\widetilde{G}_{i}^{*}$ and with the help of conditions (30) and (31), we derive that

$$
\begin{aligned}
-2 \bar{\delta} / T^{2} \geq & \lambda \widetilde{v}_{2}\left(t^{\alpha}, \tilde{x}_{2}^{\alpha}\right)+\widetilde{G}^{*}\left(\tilde{x}_{2}^{\alpha},-\widetilde{v}_{2}\left(t^{\alpha}, \tilde{x}_{2}^{\alpha}\right), \alpha\left(\tilde{x}_{1}^{\alpha}-\tilde{x}_{2}^{\alpha}\right),-X_{2}^{\alpha}\right) \\
& +\lambda \widetilde{v}_{1}\left(t^{\alpha}, \tilde{x}_{1}^{\alpha}\right)-\widetilde{G}^{*}\left(\tilde{x}_{1}^{\alpha}, \widetilde{v}_{1}\left(t^{\alpha}, \tilde{x}_{1}^{\alpha}\right), \alpha\left(\tilde{x}_{1}^{\alpha}-\tilde{x}_{2}^{\alpha}\right), X_{1}^{\alpha}\right) \\
\geq & \widetilde{G}^{*}\left(\tilde{x}_{2}^{\alpha}, \widetilde{v}_{1}\left(t^{\alpha}, \tilde{x}_{1}^{\alpha}\right), \alpha\left(\tilde{x}_{1}^{\alpha}-\tilde{x}_{2}^{\alpha}\right),-X_{2}^{\alpha}\right)-\widetilde{G}^{*}\left(\tilde{x}_{1}^{\alpha}, \widetilde{v}_{1}\left(t^{\alpha}, \tilde{x}_{1}^{\alpha}\right), \alpha\left(\tilde{x}_{1}^{\alpha}-\tilde{x}_{2}^{\alpha}\right), X_{1}^{\alpha}\right) \\
\geq & -C\left(L_{1}, L_{2}, \eta\right)\left(1+\left|\tilde{x}_{1}^{\alpha}\right|^{2}+\left|\tilde{x}_{2}^{\alpha}\right|^{2}\right)\left(\left|\widetilde{v}_{1}\left(t^{\alpha}, \tilde{x}_{1}^{\alpha}\right)\right|\left|\tilde{x}_{1}^{\alpha}-\tilde{x}_{2}^{\alpha}\right|+\alpha\left|\tilde{x}_{1}^{\alpha}-\tilde{x}_{2}^{\alpha}\right|^{2}\right) .
\end{aligned}
$$

The right-hand side tends to zero as $\alpha \rightarrow \infty$, which induces a contradiction. Consequently, we get that $\widetilde{v}^{1} \leq \widetilde{v}^{2}$, the proof is complete.

\section{References}

[1] Alvarez, O. and Bardi, M. (2003) Singular Perturbations of Nonlinear Degenerate Parabolic PDEs: a General Convergence Result. Arch. Rational Mech. Anal., 170, 17-61.

[2] Bahlalia, K., Elouaflinb, A. and Pardoux, E. (2009) Homogenization of semilinear PDEs with discontinuous averaged coefficients. Electronic Journal of Probability, 14(18), 477-499.

[3] Bahlalia, K., Elouaflinb, A. and Pardoux, E. (2017) Averaging for BSDEs with null recurrent fast component. Application to homogenization in a non periodic media. Stochastic Processes and their Applications, 127, 1321-1353.

[4] Biagini, F., Mancin, J. and Brandis, T. (2019) Robust mean-variance hedging via G-expectation. Stochastic Process. Appl., 129, 1287-1325.

[5] Buckdahn, R. and Hu, Y. (1998) Probabilistic approach to homogenizations of systems of quasilinear parabolic PDEs with periodic structures. Nonlinear Anal., 32, 609-619.

[6] Buckdahn, R., Hu, Y. and Peng, S. (1999) Probabilistic approach to homogenization of viscosity solutions of parabolic PDEs. Nonlinear Differential Equations Appl., 6, 395-411.

[7] Buckdahn, R. and Ichihara, N. (2005) Limit theorem for controlled backward SDEs and homogenization of Hamilton-Jacobi-Bellman equations. Appl. Math. Optim., 51, 1-33.

[8] Cerrai, S. and Freidlin. M. (2009) Averaging principle for stochastic reaction-diffusion equations. Probab. Theory Related Fields, 144(1-2), 137-177.

[9] Crandall, M.G., Ishii, H. and Lions, P.L. (1992) User's guide to viscosity solutions of second order partial differential equations. Bulletin of The American Mathematical Society, 27(1), 1-67.

[10] Da Prato, G. and Zabczyk, J. (1996) Ergodicity for infinite-dimensional systems. London Mathematical Society Note Series, 229, Cambridge University Press, Cambridge.

[11] Debussche, A., Hu, Y. and Tessitore, G. (2011) Ergodic BSDEs under weak dissipative assumptions. Stochastic Process. Appl., 121(3), 407-426. 
[12] Delarue, F. (2004) Auxiliary SDEs for homogenization of quasilinear PDEs with periodic coefficients. The Annals of Probability, 32(3), 2305-2361.

[13] Denis, L., Hu, M. and Peng, S. (2011) Function spaces and capacity related to a sublinear expectation: application to $G$-Brownian motion paths. Potential Anal., 34, 139-161.

[14] El Karoui, N., Kapoudjian, C., Pardoux, E., Peng, S. and Quenez, M.C. (1997) Reflected solutions of backward SDE's, and related obstacle problems for PDE's. The Annals of Probability, 23(2), 702-737.

[15] Fang, X., Peng, S., Shao, Q. and Song, Y. (2019) Limit theorems with rate of convergence under sublinear expectations. Bernoulli, 25(4A), 2564-2596.

[16] Fouque, J.-P., Pun, C. and Wong, H. (2016) Portfolio Optimization with Ambiguous Correlation and Stochastic Volatilities. SIAM J. Control Optim., 54, 2309-2338.

[17] Freidlin, M. and Wentzell, A. (1998) Random Perturbations of Dynamical Systems, 2nd Edition. Springer, New York.

[18] Fuhrman, M., Hu, Y. and Tessitore, G. (2009) Ergodic BSDEs and optimal ergodic control in Banach spaces. SIAM J. Control Optim., 48, 1542-1566.

[19] Gao, F. (2009) Pathwise properties and homomorphic flows for stochastic differential equations driven by $G$-Brownian motion. Stochastic Processes and their Applications, 119, 3356-3382.

[20] Guatteri, G. and Tessitore, G. (2021) Singular Limit of BSDEs and Optimal Control of Two Scale Stochastic Systems in Infinite Dimensional Spaces. Applied Mathematics and Optimization, 83, 1025-1051.

[21] Hu, M., Ji, S., Peng, S. and Song, Y. (2014) Backward stochastic differential equations driven by G-Brownian motion. Stochastic Process. Appl., 124, 759-784.

[22] Hu, M., Ji, S., Peng, S. and Song, Y. (2014) Comparison theorem, Feynman-Kac formula and Girsanov transformation for BSDEs driven by $G$-Brownian motion. Stochastic Process. Appl., 124, 1170-1195.

[23] Hu, M., Li, H., Wang, F. and Zheng, G. (2015) Invariant and ergodic nonlinear expectations for $G$-diffusion processes. Electronic Communications in Probability, 20, No. 30, 15pp.

[24] Hu, M. and Peng, S. (2009) On representation theorem of $G$-expectations and paths of $G$ Brownian motion. Acta Math. Appl. Sin. Engl. Ser., 25(3), 539-546.

[25] Hu, M. and Wang, F. (2018) Stochastic optimal control problem with infinite horizon driven by G-Brownian motion. ESAIM: Control, Optimisation and Calculus of Variations, 24(2), 873-899.

[26] Hu, M. and Wang, F. (2018) Ergodic BSDEs driven by G-Brownian motion and their applications. Stochastics and Dynamics, 18, No. 6, 1850050, 35pp.

[27] Hu, Y. and Madec, P.-Y. (2016) A probabilistic approach to large time behaviour of viscosity solutions of parabolic equations with Neumann boundary conditions. Applied Mathematics and Optimization, 74, 345-374.

[28] Hu, Y., Madec, P.-Y. and Richou, A. (2015) Large time behaviour of mild solutions of HamiltonJacobi-Bellman equations in infinite dimension by a probabilistic approach. SIAM J. Control Optim., 53, 378-398. 
[29] Khasminskii, R.Z. (1968) On an averaging principle for Itô stochastic differential equations. Kybernetika, Chekhoslovakia, 4(3), 260-279 (in Russian).

[30] Khasminskii, R.Z. and Krylov, N.V. (2001) On averaging principle for diffusion processes with null-recurrent fast component. Stochastic Processes and their Applications, 93, 229-240.

[31] Lin, Y., Ren, Z., Touzi, N. and Yang, J. (2020) Second order backward SDE with random terminal time. Electronic Journal of Probability, 25, No. 99, 43pp.

[32] Liu, G. (2020) Exit times for semimartingales under nonlinear expectation. Stochastic Process. Appl., 130(12), 7338-7362.

[33] Papanicolaou, G.C., Stroock, D. and Varadhan, S.R. (1977) Martingale approach to some limit theorems. Duke Univ. Math. Ser., III, 1-116.

[34] Pardoux, E. and Veretennikov, A.Y. (1999) Averaging of backward SDEs with application to semi-linear PDEs. Stoch. Stoch. Rep., 60, 255-270.

[35] Pardoux E. and Veretennikov A. Y. (2001) On the Poisson equation and diffusion approximation. I. The Annals of Probability, 29, 1061-1085.

[36] Peng, S. (2007) G-expectation, G-Brownian Motion and Related Stochastic Calculus of Itô type. Stochastic analysis and applications, 541-567, Abel Symp., 2, Springer, Berlin.

[37] Peng, S. (2008) Multi-dimensional G-Brownian motion and related stochastic calculus under $G$-expectation. Stochastic Processes and their Applications, 118(12), 2223-2253.

[38] Peng, S. (2019) Nonlinear expectations and stochastic calculus under uncertainty. Springer-Verlag Berlin Heidelberg.

[39] Possamaï, D., Tan, X. and Zhou, C. (2018) Stochastic control for a class of nonlinear kernels and applications. The Annals of Probability, 46(1), 551-603.

[40] Röckner, M. and Xie, L. (2021) Diffusion approximation for fully coupled stochastic differential equations. The Annals of Probability, 49(3), 1205-1236.

[41] Soner, H.M., Touzi, N. and Zhang, J. (2011) Martingale representation theorem for the Gexpectation. Stochastic Process. Appl., 121, 265-287.

[42] Soner, H.M., Touzi, N. and Zhang, J. (2012) Wellposedness of Second Order Backward SDEs. Probability Theory and Related Fields, 153, 149-190.

[43] Song, Y. (2021) Gradient Estimates for Nonlinear Diffusion Semigroups by Coupling Methods. Science China Mathematics, 64, 1093-1108.

[44] Song, Y. (2019) Properties of $G$-martingales with finite variation and the application to $G$-Sobolev spaces. Stochastic Process. Appl., 129(6), 2066-2085. 